\title{
LARGE DEVIATIONS FOR NON-UNIFORMLY EXPANDING MAPS
}

\author{
V. ARAÚJO AND M. J. PACIFICO
}

\begin{abstract}
We obtain large deviation bounds for non-uniformly expanding maps with nonflat singularities or criticalities and for partially hyperbolic non-uniformly expanding attracting sets. That is, given a continuous function we consider its space average with respect to a physical measure and compare this with the time averages along orbits of the map, showing that the Lebesgue measure of the set of points whose time averages stay away from the space average tends to zero exponentially fast with the number of iterates involved. As easy by-products we deduce escape rates from subsets of the basins of physical measures for these types of maps. The rates of decay are naturally related to the metric entropy and pressure function of the system with respect to a family of equilibrium states. The corrections added to the published version of this text appear in bold; see last section for a list of changes.
\end{abstract}

\section{CONTENTS}

1. Introduction

1.1. Statement of the results

1.2. Partially hyperbolic diffeomorphisms

1.3. Escape rates

1.4. Comments and organization of the paper 9

2. Examples of application 10

2.1. Quadratic maps and infinite-modal maps 10

2.2. Piecewise smooth one-dimensional expanding maps 10

2.3. Non-uniformly expanding local diffeomorphisms 11

2.4. Viana maps 11

2.5. Partially hyperbolic non-uniformly expanding diffeomorphisms 11

3. Hyperbolic times 12

3.1. Coverings by hyperbolic preballs 13

3.2. The partially hyperbolic setting 18

3.3. The volume of dynamical balls 21

4. Hyperbolic times and large deviations 23

4.1. Upper bound for local diffeomorphisms 23

4.2. Upper bound for partially hyperbolic diffeomorphisms 25

4.3. Upper bound with singular/critical set 25

5. Strictly negative upper bound 28

Date: August 30, 2021.

2000 Mathematics Subject Classification. 37D25, 37A50, 37B40, 37C40.

Key words and phrases. non-uniform expansion, physical measures, hyperbolic times, large deviations.

V.A. was partially supported by CNPq-Brazil and FCT-Portugal through CMUP and POCI/MAT/61237/2004. M.J.P. was partially supported by CNPq-Brazil/FAPERJ-Brazil/Pronex Dynamical Systems. 
5.1. The local diffeomorphism and partially hyperbolic case 28

5.2. The case with singular/critical set $\quad 29$

5.3. Ruelle's Inequality for maps with non-flat singularities 30

References 34

6. List of changes with respect to the published version 36

\section{INTRODUCTION}

Smooth Ergodic Theory provides asymptotic information on the behavior of a dynamical system, given by a smooth transformation, when times goes to infinity. This statistical approach to Dynamics has provided valuable insights into many phenomena: from the remarkable result of Jakobson [33] (see also [13, 14]) showing the existence of many (positive Lebesgue measure of) parameters $a \in(0,2)$ for which the corresponding map of the quadratic family $x \mapsto a-x^{2}$ has positive Lyapunov exponent along almost every orbit; to the study of higher dimensional systems: related ideas provided the first clue to the nature of the Hénon attractor $[14,42]$ or the existence of robust classes of maps which are not uniformly expanding but exhibit several distinct positive Lyapunov exponents [59], and enabled one to understand the statistical properties of these and other classes of systems [46, 15, 63, 17, 2, 19, 6].

The basic ideas can be traced back to the Boltzmann Ergodic Hypothesis from Statistical Mechanics which was the main motivation behind the celebrated Birkhoff's Ergodic Theorem ensuring the equality between temporal and spatial averages with respect to a (ergodic) probability measure $\mu$ invariant under a measurable transformation $f: M \rightarrow M$ of a compact manifold $M$, i.e. for every continuous map $\varphi: M \rightarrow \mathbb{R}$ we have

$$
\lim _{n \rightarrow+\infty} \frac{1}{n} \sum_{j=0}^{n-1} \varphi\left(f^{j}(x)\right)=\int \varphi d \mu
$$

for $\mu$ almost every point $x \in M$. Defining $B(\mu)$, the ergodic basin of $\mu$, to be the set of points for which (1.1) holds for every continuous function $\varphi$, the Ergodic Theorem says that $\mu(B(\mu))=1$ for all ergodic $f$-invariant probability measures $\mu$. Since ergodic measures can be, for instance, Dirac masses concentrated on periodic orbits, the Ergodic Theorem in itself does not always provide information about the asymptotic behavior of "big" subsets of points. The notion of "big" can arguably be taken as meaning "having positive Lebesgue measure (or positive volume)", since such sets are in principle "observable sets" when interpreting $f: M \rightarrow M$ as a model of physical, biological or economic phenomena. Correspondingly invariant probability measures $\mu$ for which $B(\mu)$ has positive volume are called physical (or Sinai-Ruelle-Bowen) measures.

This kind of measures was first constructed for (uniformly) hyperbolic diffeomorphisms by Sinai, Ruelle and Bowen $[57,51,21]$. Such measures for non-uniformly hyperbolic maps where obtained more recently $[46,15,16,2]$.

We say that a local diffeomorphism $f$ of a compact manifold is (uniformly) expanding if there exists $n \geq 1$ such that for all $x$ and every tangent vector $v$ at $x$

$$
\left\|D f^{n}(x) v\right\| \geq 2\|v\| .
$$

For diffeomorphisms of compact manifolds, the notion of hyperbolicity requires the existence of two complementary directions given by two (continuous) subbundles $E$ and $F$ of the tangent 
bundle admitting $n \geq 1$ such that for all points $x$ and tangent vectors $(u, v) \in E_{x} \oplus F_{x}$

$$
\left\|D f^{n}(x) u\right\| \leq \frac{1}{2}\|u\| \quad \text { and } \quad\left\|D f^{n}(x) v\right\| \geq 2\|v\| .
$$

The probabilistic properties of physical measures are an object of intense study, see e.g. $[21,63,17,3,4,7,30,11]$. The leitmotif is that the sequence $\left\{\varphi \circ f^{n}\right\}_{n \geq 0}$ should behave like an i.i.d. random variable, at least asymptotically.

Here we are concerned with the rate of convergence of the time averages (1.1) for nonuniformly expanding maps (NUE) and partially hyperbolic non-uniformly expanding diffeomorphisms (PHNUE), where condition (1.2) and the right hand side condition of (1.3) are replaced by the following asymptotic ones

NUE: for Lebesgue almost every point $x$ there exists $n=n(x) \geq 1$ such that $\left\|D f^{n}(x) v\right\| \geq$ $2\|v\|$ for all vector $v \in T_{x} M$;

PHNUE: for Lebesgue almost all points $x$ there exists $n=n(x) \geq 1$ such that $\left\|D f^{n}(x) v\right\| \geq 2\|v\|$ for all vector $v \in F_{x}$.

We note that if conditions NUE or PHNUE hold for every point then the system is uniformly expanding or uniformly hyperbolic $[58,5]$. We also consider transformations which are diffeomorphisms outside a "small" (zero volume) set of singular or critical points such that the orbits of Lebesgue almost all points have slow recurrence near this singular set. For more details see the statement of results below.

The question of the speed of convergence to equilibrium arises naturally from so-called thermodynamical formalism of (uniformly) hyperbolic diffeomorphisms, borrowed from statistical mechanics by Ruelle, Sinai and Bowen (among others, see e.g. [20, 53, 54, 29, 18]) through the dictionary between one-dimensional lattices and (uniformly) expanding maps (Gibbs distributions and equilibrium states in particular) provided by the existence of a finite Markov partition for the latter systems. Indeed chaotic dynamics is associated with loss of memory and creation of information (two views of the same phenomenon) as the system evolves. These notions are formalized in a variety of ways, from entropy, the exponential rate of creation of information; to decay of correlations, which measures the speed the system "forgets" its initial state; through large deviations results, which measure how fast the system approaches a state of equilibrium after evolving from almost every initial state. However, even with abundance of positive Lyapunov exponents, which is the essential content of the non-uniform expansion/hyperbolicity conditions above, extending this theory from uniform to the non-uniform hyperbolic setting demands considering (if one is optimistic), through the dictionary already mentioned, Markov partitions with infinitely many symbols leading to a thermodynamical formalism of gases with infinitely many states, a hard subject not yet well understood (see e.g. [24, 11] for recent developments).

Assuming conditions NUE or PHNUE we are able to extend some of the large deviation results for uniformly hyperbolic system in $[62,35]$ (see also $[27,28]$ for sharp estimates though a different approach) and strengthen, in a definite sense, the idea that non-uniformly hyperbolic systems are chaotic: they satisfy a version of the classical large deviation results for i.i.d. random variables. More precisely, if we set $\delta>0$ as an acceptable error margin and consider

$$
B_{n}=\left\{x \in M:\left|\frac{1}{n} \sum_{j=0}^{n-1} \varphi\left(f^{j}(x)\right)-\int \varphi d \mu\right|>\delta\right\}
$$


then we are able to ascertain whether the Lebesgue measure of $B_{n}$ decays to zero exponentially fast, i.e. weather there are constants $C, \xi>0$ such that

$$
\operatorname{Leb}\left(B_{n}\right) \leq C e^{-\xi n} \quad \text { for all } n \geq 1 .
$$

The values of $C, \xi>0$ above depend on $\delta, \varphi$ and on global invariants for the map $f$ such as the metric entropy and the pressure function of $f$ with respect to some equilibrium measures, as detailed in the next section.

We are able to obtain large deviation rates as in (1.4) for non-uniformly expanding local diffeomorphisms and also for endomorphisms and maps with non-flat singularities and critical points under a condition on the rate of approximation of most orbits to the critical/singular set. In particular we are able to obtain an exponential decay rate as above for piecewise expanding maps with infinitely many smoothness domains, for quadratic maps corresponding to a positive Lebesgue measure subset of parameters and for a class of maps with infinitely many critical points. Moreover we also obtain the same kind of rates for partially hyperbolic attracting sets with a non-uniformly expanding direction.

1.1. Statement of the results. We denote by $\|\cdot\|$ a Riemannian norm on the compact boundaryless manifold $M$, by $d$ the induced distance and by Leb a Riemannian volume form, which we call Lebesgue measure or volume and assume to be normalized: $\operatorname{Leb}(M)=1$.

We start by describing one of the class of maps that we are going to consider. Let $f: M \rightarrow$ $M$ be a map of the compact manifold $M$ which is a $C^{2}$ local diffeomorphism outside a set $\mathcal{S} \subset M$ with zero Lebesgue measure. We assume that $f$ behaves like a power of the distance close to $\mathcal{S}$ : there are constants $B>1$ and $\beta>0$ for which

(S1) $\frac{1}{B} d(x, \mathcal{S})^{\beta} \leq \frac{\|D f(x) v\|}{\|v\|} \leq B d(x, \mathcal{S})^{-\beta}$;

$$
\begin{aligned}
& \left|\log \left\|D f(x)^{-1}\right\|-\log \left\|D f(y)^{-1}\right\|\right| \leq B \frac{d(x, y)}{d(x, \mathcal{S})^{\beta}} \\
& |\log | \operatorname{det} D f(x)^{-1}|-\log | \operatorname{det} D f(y)^{-1}|| \leq B \frac{d(x, y)}{d(x, \mathcal{S})^{\beta}}
\end{aligned}
$$

for every $x, y \in M \backslash \mathcal{S}$ with $d(x, y)<d(x, \mathcal{S}) / 2$ and $v \in T_{x} M \backslash\{0\}$. The singular set $\mathcal{S}$ may be thought of as containing those points $x$ where $D f(x)$ is either not defined or else is non-invertible. Note in particular that $\mathcal{S}$ contains the set $\mathcal{C}$ of critical points of $f$, i.e. the set of points (which may be empty) where $D f(x)$ is not invertible. We refer to this kind of singular sets as non-flat since conditions (S1) to (S3) above are natural generalizations to arbitrary dimensions of the notion of non-flat critical point from one-dimensional dynamics, see e.g.[26].

In what follows we write $S_{n} \varphi(x)$ for $\sum_{i=0}^{n-1} \varphi\left(f^{i}(x)\right)$ and a function $\varphi: M \rightarrow \mathbb{R}$. We say that $f$ as above is non-uniformly expanding if there exists $c>0$ such that

$$
\limsup _{n \rightarrow+\infty} \frac{1}{n} S_{n} \psi(x) \leq-c \quad \text { where } \quad \psi(x)=\log \left\|D f(x)^{-1}\right\|,
$$

for Lebesgue almost every $x \in M$. We need to control the rate of approximation of most orbits to the singular set. We say that $f$ has slow recurrence to the singular set $\mathcal{S}$ if for every $\varepsilon>0$ there exists $\delta>0$ such that

$$
\limsup _{n \rightarrow \infty} \frac{1}{n} S_{n} \Delta_{\delta}(x)<\varepsilon \quad \text { with } \quad \Delta_{\delta}(x)=\left|\log d_{\delta}(x, \mathcal{S})\right|
$$


for Lebesgue almost every $x \in M$, where for any given $\delta>0$ we define the smooth $\delta$-truncated distance from $x \in M$ to $\mathcal{S}$ by

$$
d_{\delta}(x, \mathcal{S})=\xi_{\delta}(d(x, \mathcal{S})) \cdot d(x, \mathcal{S})+1-\xi_{\delta}(d(x, \mathcal{S}))
$$

where $\xi_{\delta}: \mathbb{R} \rightarrow[0,1]$ is a standard $C^{\infty}$ auxiliary function satisfying

$$
\xi_{\delta}(x)=1 \text { if }|x| \leq \delta \text { and } \xi_{\delta}(x)=0 \text { if }|x| \geq 2 \delta .
$$

Observe that $\Delta_{\delta}$ is non-negative and continuous away from $\mathcal{S}$ and identically zero $2 \delta$-away from $\mathcal{S}$.

These notions where presented in [6] for higher dimensional maps abstracted from similar notions from one-dimensional maps [26] and previous work on maps with singularities [34], and in $[6,1]$ the following result on existence of finitely many physical measures was obtained.

Theorem 1.1. Let $f: M \rightarrow M$ be a $C^{2}$ local diffeomorphism outside a non-flat singular set $\mathcal{S}$. Assume that $f$ is non-uniformly expanding with slow recurrence to $\mathcal{S}$. Then there are finitely many physical (or Sinai-Ruelle-Bowen) measures $\mu_{1}, \ldots, \mu_{k}$ whose basins cover the manifold Lebesgue almost everywhere, that is $B\left(\mu_{1}\right) \cup \cdots \cup B\left(\mu_{k}\right)=M, \quad$ Leb- $\bmod 0$.

We say that $f$ is a regular map if $f_{*}$ Leb $\ll$ Leb, that is, if $E \subset M$ is such that $\operatorname{Leb}(E)=0$, then Leb $\left(f^{-1}(E)\right)=0$. We denote by $\mathcal{M}_{f}$ the family of all invariant probability measures with respect to $f$, by $\mathcal{M}_{f}^{e}$ the family of all ergodic $f$-invariant probability measures, and define

$$
B(x, n, \varepsilon)=\left\{y \in M: d\left(f^{i}(x), f^{i}(y)\right)<\varepsilon, i=0, \ldots, n-1\right\}
$$

the $(n, \varepsilon)$-dynamical ball around $x \in M$. Large deviation statements are usually related to local entropies which originated from the works of Shannon, McMillan and Breiman [56, 40, 22] and can be succinctly expressed as follows on a metric space after the work of Brin and Katok [23]. For any finite Borel measure $m$ on $M$ define its local entropy at $x$ to be

$$
h_{m}(f)(x)=\lim _{\varepsilon \rightarrow 0} \limsup _{n \rightarrow \infty}-\frac{1}{n} \log m(B(x, n, \varepsilon)) .
$$

In [23] it is proved that this limit exists $m$-almost everywhere whenever $m$ is a $f$-invariant probability measure. The metric (or measure-theoretic) entropy of the map $f$ is then defined to be the non-negative number

$$
h_{m}(f)=\int h_{m}(f)(x) d m(x) .
$$

Moreover the function $h_{m}(f)(x)$ is $f$-invariant, so it is almost everywhere constant if $m$ is $f$-ergodic.

We will be interested in the case $m=$ Lebesgue measure (volume) on $M$, which is usually not an invariant measure in our setting and for $\nu \in \mathcal{M}_{f}$ we consider

$$
h_{m}(f, \nu)=\nu-\operatorname{ess} \sup h_{m}(f) .
$$

Note that given $\nu \in \mathcal{M}_{f}$ the value of $h_{\nu}(f)$ is not at all related to $h_{\text {Leb }}(f, \nu)$, unless both measures coincide and $\nu \in \mathcal{M}_{f}^{e}$, in which case $h_{\nu}(f, \nu)=h_{\nu}(f)$.

Theorem A. Let $f: M \rightarrow M$ be a regular $C^{1+\alpha}$ local diffeomorphism outside a non-flat singular set $\mathcal{S}$, for some $\alpha \in(0,1)$. Assume that $f$ is non-uniformly expanding with slow recurrence to $\mathcal{S}$. Then writing $J=\log |\operatorname{det} D f|$, given $c \in \mathbb{R}$ and a continuous function $\varphi: M \rightarrow \mathbb{R}$ 
(1) if $h_{\mathrm{top}}(f)<\infty$, then

$$
\begin{aligned}
\liminf _{n \rightarrow+\infty} \frac{1}{n} \log \operatorname{Leb}\left(\left\{x \in M: \frac{1}{n} S_{n} \varphi(x)>c\right\}\right) \\
\geq \sup \left\{h_{\nu}(f)-h_{\mathrm{Leb}}(f, \nu): \nu \in \mathcal{M}_{f}^{e}, \int \varphi d \nu>c\right\} ;
\end{aligned}
$$

(2) if $\mathcal{S}=\emptyset$ ( $f$ is a local diffeomorphism) then

$$
\begin{aligned}
\limsup _{n \rightarrow+\infty} & \frac{1}{n} \log \operatorname{Leb}\left(\left\{x \in M: \frac{1}{n} S_{n} \varphi(x) \geq c\right\}\right. \\
\leq & \sup \left\{h_{\nu}(f)-\int J d \nu: \nu \in \mathcal{M}_{f}, \int \varphi d \nu \geq c\right\} .
\end{aligned}
$$

(3) in general for any given $\eta>0$ there exists $\varepsilon, \delta>0$ such that

$$
\begin{aligned}
\limsup _{n \rightarrow+\infty} & \frac{1}{n} \log \operatorname{Leb}\left(\left\{x \in M: \frac{1}{n} S_{n} \varphi(x) \geq c \text { and } \frac{1}{n} S_{n} \Delta_{\delta}(x) \leq \varepsilon\right\}\right) \\
& \leq \eta+\sup \left\{h_{\nu}(f)-\int J d \nu: \nu \in \mathcal{M}_{f}, \int \varphi d \nu \geq c \text { and } \Delta_{\delta} \in L^{1}(\nu)\right\} .
\end{aligned}
$$

We say that a measure $\nu \in \mathcal{M}_{f}$ is an equilibrium state for $f$ with respect to $J$ (or just an equilibrium state in what follows) if

$$
h_{\nu}(f)=\nu(J)=\int J d \nu
$$

As the above statement shows, equilibrium states are involved in the determination of the asymptotic rates of deviation. Given $\varepsilon, \delta>0$ we write $\mathbb{E}=\mathbb{E}_{\varepsilon, \delta}$ for the family of all equilibrium states $\mu$ of $f$ with respect to $J$ such that $\mu\left(\Delta_{\delta}\right) \leq \varepsilon$ and, given a continuous $\varphi: M \rightarrow \mathbb{R}$, we define $\mathbb{E}(\varphi)=\{\nu(\varphi): \nu \in \mathbb{E}\}$.

Remark 1.2. Note that the expressions obtained in items (1) and (2) of the statement of Theorem A are not comparable since the supremum is taken over all invariant measures in item (2), while we consider only ergodic invariant measures in item (1).

We say that $\mu \in \mathcal{M}_{f}$ is a weak expanding measure if the subset of points weakly satisfying (1.5) has full $\mu$-measure, that is

$$
\mu\left\{x \in M: \limsup _{n \rightarrow+\infty} \frac{1}{n} S_{n} \psi(x) \leq 0\right\}=1 .
$$

We are able to deduce that the supremum in the statement of Theorem A is strictly negative for non-uniformly expanding maps with slow recurrence to the singular set such that all equilibrium states $\mathbb{E}$ are expanding measures.

Theorem B. Let $f: M \rightarrow M$ be a local diffeomorphism outside a non-flat singular set $\mathcal{S}$ which is non-uniformly expanding, has slow recurrence to $\mathcal{S}$ and every element in $\mathbb{E}$ is weak expanding. For $\omega>0$ and a continuous function $\varphi: M \rightarrow \mathbb{R}$ there exists $\varepsilon, \delta>0$ arbitrarily close to 0 such that, writing

$$
A_{n}=\left\{x \in M: \frac{1}{n} S_{n} \Delta_{\delta}(x) \leq \varepsilon\right\}
$$


and

$$
B_{n}=\left\{x \in M: \inf \left\{\left|\frac{1}{n} S_{n} \varphi(x)-\eta(\varphi)\right|: \eta \in \mathbb{E}\right\}>\omega\right\}
$$

we get

$$
\limsup _{n \rightarrow+\infty} \frac{1}{n} \log \operatorname{Leb}\left(A_{n} \cap B_{n}\right)<0 .
$$

Clearly if $\mathcal{S}=\emptyset$ ( $f$ is a local diffeomorphism) then $A_{n}=M$ and we obtain an asymptotic large deviation rate for the sets $B_{n}$. Otherwise to get a similar upper bound for $\operatorname{Leb}\left(B_{n}\right)$ we need an extra assumption on the decay of the measure of the tail sets $M \backslash A_{n}$.

Corollary C. In the setting of Theorem $B$ with $\mathcal{S} \neq \emptyset$, if $f$ also satisfies

$$
\limsup _{n \rightarrow \infty} \frac{1}{n} \log \operatorname{Leb}\left(M \backslash A_{n}\right)<0
$$

then we have also

$$
\limsup _{n \rightarrow \infty} \frac{1}{n} \log \operatorname{Leb}\left(B_{n}\right)<0
$$

Remark 1.3. Observe that if $\mu$ is a $f$-ergodic absolutely continuous probability measure whose support is the entire manifold, then the slow recurrence condition (1.6) is the same as saying that $\log d(x, \mathcal{S})$ is $\mu$-integrable.

Note that for any $C^{2}$ endomorphism $f$ (i.e. the singular set $\mathcal{S}$ of $f$ coincides with the critical set $\mathcal{C}$ of $f$ ) we have $|\log d(x, \mathcal{C})| \geq \Delta_{\delta}(x)$ and, as shown in [37], the function $\log d(x, \mathcal{C})$ is $\mu$ integrable for every $f$-invariant probability measure. However we need to deal with families of invariant probability measures for which $\log d(x, \mathcal{C})$ is uniformly integrable so that the proofs of Theorems A and B can be carried out with our arguments. This is why we need the sets $A_{n}$ in the previous statements. To the best of our knowledge no such general integrability result for $\log d(x, \mathcal{S})$ exists with respect to invariant probability measures for maps with non-flat singularities.

1.2. Partially hyperbolic diffeomorphisms. Let now $f: M \rightarrow M$ be a $C^{2}$ diffeomorphism. We say that a compact $f$-invariant set $\Lambda$ is an attracting set if it admits a trapping region, that is, an open neighborhood $U \supset \Lambda$ such that $\overline{f(U)} \subset U$ and $\Lambda=\cap_{n \geq 0} f^{n}(U)$. Note that we may have $\Lambda=U=M$ (where $M$ is connected).

As shown in [62], for every attracting set $\Lambda$ and every physical probability measure $\nu$ supported in $\Lambda$, given $\delta>0$ and a continuous $\varphi: \bar{U} \rightarrow \mathbb{R}$ we have

$$
\begin{aligned}
\liminf _{n \rightarrow \infty} \frac{1}{n} \log \operatorname{Leb}\left\{\left|\frac{1}{n} S_{n} \varphi-\int \varphi d \mu\right|>\delta\right\} \geq \\
\quad \sup \left\{h_{\nu}(f)-\int \Sigma^{+} d \nu: \nu \in \mathcal{M}_{f}^{e},\left|\int \varphi d \nu-\int \varphi d \mu\right| \geq \delta\right\} .
\end{aligned}
$$

Here $\Sigma^{+}$denotes the sum of the positive Lyapunov exponents at a given point of $M$. Recall that Ruelle's Inequality $h_{\mu}(f) \leq \int \Sigma^{+} d \mu$ is true of every $C^{1}$-diffeomorphism [52].

An attracting set $\Lambda$ is partially hyperbolic (see e.g. [46, 18]) if there exists a continuous splitting $E \oplus F$ of the tangent bundle of $M$ over $\Lambda$ along two complementary vector subbundles satisfying

$$
\text { - } D f \text {-invariance: } D f\left(E_{x}\right)=E_{f(x)} \text { and } D f\left(F_{x}\right)=F_{f(x)} \text { for all } x \in \Lambda \text {; }
$$


- domination: there exists $n \geq 1$ such that

$$
\left\|D f^{n} \mid E_{x}\right\| \cdot\left\|\left(D f^{n} \mid F_{x}\right)^{-1}\right\| \leq \frac{1}{2} \text { for all } \quad x \in \Lambda ;
$$

- $E$ is uniformly contracting: there is $n \geq 1$ such that $\left\|D f^{n} \mid E_{x}\right\| \leq \frac{1}{2}$ for all $x \in \Lambda$.

In this setting we denote by $J$ the logarithm of the Jacobian along the centre-unstable direction $J(x)=\log |\operatorname{det} D f| F_{x} \mid$ and by $\mathbb{E}$ the family of all equilibrium states with respect to $J$, i.e. the set of all $f$-invariant probability measures $\nu$ such that $h_{\nu}(f)=\nu(J)$.

We will assume further that the $F$ direction only has positive Lyapunov exponents in the following sense, introduced in [6]. We say that a partially hyperbolic attractor with trapping region $U$ is non-uniformly expanding if there exists $c>0$ such that

$$
\limsup _{n \rightarrow \infty} \frac{1}{n} \sum_{j=0}^{n-1} \log \left\|\left(D f \mid F_{f^{j}(x)}\right)^{-1}\right\| \leq-c
$$

for Lebesgue almost every point $x \in U$. In [6] the following was obtained.

Theorem 1.4. Let $\Lambda$ be a partially hyperbolic non-uniformly expanding attracting set for a $C^{2}$ diffeomorphism $f$ with trapping region $U$. Then there are finitely many equilibrium states which are physical measures supported in $\Lambda$, and whose basins cover $U$ except for a subset of zero Lebesgue measure.

We say that a measure $\mu \in \mathcal{M}_{f}$ supported in $U$ is weak expanding if the subset of points satisfying a weak non-uniformly expanding condition has full $\mu$-measure, that is

$$
\mu\left\{x \in U: \limsup _{n \rightarrow \infty} \frac{1}{n} \sum_{j=0}^{n-1} \log \left\|\left(D f \mid F_{f^{j}(x)}\right)^{-1}\right\| \leq 0\right\}=1 .
$$

We are able to obtain an upper bound entirely analogous to item 2 of Theorem A replacing $M$ by the points in the trapping region $U$ of a partially hyperbolic non-uniformly expanding attracting set $\Lambda$ for a $C^{2}$ diffeomorphism. Then for the same kind of attracting sets we obtain an upper bound for the subset corresponding to (1.7).

Theorem D. Let $f: M \rightarrow M$ be a $C^{2}$ diffeomorphism exhibiting a partially hyperbolic nonuniformly expanding attracting set $\Lambda$ with isolating neighborhood $U \supset \Lambda$ such that every measure in $\mathbb{E}$ is weak expanding. Given $\omega>0$ and a continuous $\varphi: \bar{U} \rightarrow \mathbb{R}$, define

$$
B_{n}=\left\{x \in U: \inf \left\{\left|\frac{1}{n} S_{n} \varphi(x)-\eta(\varphi)\right|: \eta \in \mathbb{E}\right\}>\omega\right\} .
$$

Then

$$
\limsup _{n \rightarrow \infty} \frac{1}{n} \log \operatorname{Leb}\left(B_{n}\right)<0 .
$$

1.3. Escape rates. Using the estimates obtained above and the observation that for any compact subset $K$ and a given $\varepsilon>0$ we can find an open set $W \supset K$ and a continuous function $\varphi: M \rightarrow \mathbb{R}$ such that

- $\operatorname{Leb}(W \backslash K)<\varepsilon$;

- $0 \leq \varphi \leq 1, \varphi \mid K \equiv 1$ and $\varphi \mid(M \backslash W) \equiv 0$, 
we see that for $n \geq 1$

$$
\left\{x \in K: f(x) \in K, \ldots, f^{n-1}(x) \in K\right\} \subset\left\{x \in M: \frac{1}{n} S_{n} \varphi(x) \geq 1\right\}
$$

and so we get the following (recall the definition of $A_{n}$ in the statement of Theorem B).

Corollary E. Let $f: M \rightarrow M$ be a local diffeomorphism outside a non-flat singular set $\mathcal{S}$ which is non-uniformly expanding, has slow recurrence to $\mathcal{S}$ and every measure in $\mathbb{E}$ is weak expanding. Let $K$ be a compact subset such that $\mu(K)<1$ for all $\mu$ in the weak*closure $\overline{\mathbb{E}}$ of $\mathbb{E}$. Then for a pair $\varepsilon, \delta>0$ close to 0

$$
\limsup _{n \rightarrow+\infty} \frac{1}{n} \log \operatorname{Leb}\left(\left\{x \in K \cap A_{n}: f^{j}(x) \in K, j=1, \ldots, n-1\right\}\right)<0 .
$$

Moreover if $\lim \sup _{n \rightarrow \infty} \frac{1}{n} \log \operatorname{Leb}\left(M \backslash A_{n}\right)<0$ then

$$
\limsup _{n \rightarrow+\infty} \frac{1}{n} \log \operatorname{Leb}\left(\left\{x \in K, f(x) \in K, \ldots, f^{n-1}(x) \in K\right\}\right)<0 .
$$

In the setting of a partially hyperbolic non-uniformly expanding attracting set we get, using the same reasoning as above

Corollary F. Let $f: M \rightarrow M$ be a diffeomorphism and $\Lambda$ a partially hyperbolic non-uniformly expanding attracting set with isolating neighborhood $U$ such that every measure in $\mathbb{E}$ is weak expanding. Let $K \subset U$ be a compact subset such that $\mu(K)<1$ for all $\mu$ in the weak $k^{*}$-closure $\overline{\mathbb{E}}$ of $\mathbb{E}$. Then

$$
\limsup _{n \rightarrow+\infty} \frac{1}{n} \log \operatorname{Leb}\left(\left\{x \in K, f(x) \in K, \ldots, f^{n-1}(x) \in K\right\}\right)<0 .
$$

1.4. Comments and organization of the paper. All the arguments use in fact that $f$ is $C^{1}$ and that its derivative $D f$ is $\alpha$-Hölder continuous with respect to the fixed Riemannian norm on $M$, so that all we need is $f$ to be a $C^{1+\alpha}$ local diffeomorphism outside the singular set, for some $\alpha \in(0,1)$.

The difficulties we face when considering transformations which are not uniformly hyperbolic and present singularities are related to the construction of the measures $\nu$, appearing in the supremum at item (1) of the statement of Theorem A, as a weak* limit of discrete measures which converge to an invariant measure and are supported on the set one wishes to control. Since we need to take weak* limits of measures against discontinuous test functions, the main body of work in this paper is to provide sufficient estimates for convergence imposing some conditions on the dynamics of the maps involved.

The existence of a lower bound for the large deviation rate with the same expression as in items (2) and (3) of the statement of Theorem A depends on the existence and uniqueness of equilibrium states (the reader should see [35] for precise statements and also for counterexamples when uniqueness is not satisfied). However existence and uniqueness of equilibrium states for non-uniformly expanding maps is still an open problem for most potentials in spite of recent progress in this direction by several authors, see e.g. [44, 12, 11].

Recently Pinheiro [47] has extended the statement of Theorem 1.1 replacing the limsup in condition (1.5) by liminf, keeping the same conclusions involving the existence of finitely many physical measures and of a positive density of hyperbolic times Lebesgue almost everywhere. Hence our statements are automatically valid in this more general setting. 
In what follows, we start by presenting some non-trivial classes of maps to which our results are applicable, in Section 2. In Section 3 we present preliminary technical results to be used in the following sections. Theorem A is then proved in Subsection 4.1 for local diffeomorphisms, in Subsection 4.2 for partially hyperbolic non-uniformly expanding diffeomorphisms and in Subsection 4.3 for maps with singularities or criticalities. In Section 5 we deduce Theorem B from Theorem A, first for local diffeomorphisms and for the partially hyperbolic case in Subsection 5.1, and then with singularities or criticalities in Subsection 5.2, together with an extension of Ruelle's Inequality to maps with non-flat singularities in Subsection 5.3.

Acknowledgements. We are thankful to M. Viana (IMPA) for valuable comments and suggestions during the elaboration of this text. Later, P. Varandas (UFBA) pointed out to the authors several issues with the proofs that prompted the preparation of this corrected version. The authors are also indebted to the fine scientific environment and access to the superb mathematical library of IMPA during the preparation of the earliest versions of the manuscript.

\section{EXAMPLES OF APPLICATION}

Here we show that there are many examples of maps in the conditions of Theorem B, Corollary C or Theorem D.

2.1. Quadratic maps and infinite-modal maps. In [9] the following $C^{\infty}$ family of maps of $I=[-1,1]$ with infinitely many critical points was considered:

$$
f_{\mu}(z)= \begin{cases}f(z)+\mu & \text { for } z \in(0, \varepsilon] \\ f(z)-\mu & \text { for } z \in[-\varepsilon, 0)\end{cases}
$$

where $f: I \rightarrow I$ is an expanding extension of

$$
\hat{f}:[-\varepsilon, \varepsilon] \rightarrow[-1,1], \quad \hat{f}(z)= \begin{cases}\left.a z^{\alpha} \sin (\beta \log (1 / z))\right) & \text { if } z>0 \\ \left.-a|z|^{\alpha} \sin (\beta \log (1 /|z|))\right) & \text { if } z<0,\end{cases}
$$

to $I$ (i.e. $\left|f^{\prime}\right| \gg 1$ on $I \backslash[-\varepsilon, \varepsilon]$ ), with $a>0,0<\alpha<1, \beta>0$ and $\varepsilon>0$. It was shown that there exists a positive Lebesgue measure subset $P$ of parameters in $(-\varepsilon, \varepsilon)$ such that for $\mu \in P$ the map $f_{\mu}$ is non-uniformly expanding and has slow recurrence to the non-flat infinite and denumerable singular set. Moreover for the same parameters the decay rate of the tail set is exponential, i.e. (1.9) is true. If all equilibrium states with respect to $-\log \left|f^{\prime}\right|$ are weak expanding, then $f_{\mu}$ for $\mu \in P$ is in the setting of Corollaries $\mathrm{C}$ and $\mathrm{E}$.

Analogous results hold for the quadratic family $Q_{a}(x)=a-x^{2}$ (and also for general $C^{2}$ unimodal families), so that Corollaries $\mathrm{C}$ and $\mathrm{E}$ apply to quadratic maps for a positive Lebesgue measure subset of parameters since all invariant measures are weak expanding in this setting; see [49].

2.2. Piecewise smooth one-dimensional expanding maps. Let $f: I \rightarrow I$ be a map admitting a sequence $\mathcal{S}=\left\{a_{n}, n \geq 1\right\} \subset I=[-1,1]$ such that for every connected component $G$ of $I \backslash \mathcal{S}$ we have that $f \mid G$ is $C^{1}$ diffeomorphism with its image and there exists $n \in \mathbb{Z}^{+}$ so that $\left|D f^{n}(x)\right|>1$ for all $x \in I \backslash \cup_{i=0}^{n-1} f^{-i} \mathcal{S}$. Assume that $\mathcal{S}$ is a non-flat singular set for $f$ and that $f$ admits a absolutely continuous ergodic invariant probability measure $\mu$ with positive Lyapunov exponent and such that $\log d(x, \mathcal{S})$ is $\mu$-integrable and $\operatorname{supp} \mu=I$. Then $f$ is in the setting of Theorem B since all invariant measures are weak expanding in this case. 
Examples of this kind of maps are the Gauss map [60], and transitive piecewise one dimensional maps satisfying the conditions in [55] (see also [60]), that is there exists $\kappa>0$ such that for every connected component $G$ of $I \backslash \mathcal{S}$ we also have

$$
\operatorname{var}_{G} \frac{1}{\left|f^{\prime}\right|} \leq \kappa \cdot \sup _{G} \frac{1}{\left|f^{\prime}\right|} \text { and } \quad \sum_{G} \sup _{G} \frac{1}{\left|f^{\prime}\right|} \leq \kappa .
$$

More concrete examples are Lorenz-like maps [36, 60] (even with criticalities [38]) and the maps introduced by Rovella [50, 41].

A proof of the exponential decay of the tail set for this class of maps is not available in the literature to the best of our knowledge but can be done as an application of the technique of exclusion of parameters introduced in [13] (the details will appear in forthcoming work [8]), so that Corollaries $\mathrm{C}$ and $\mathrm{E}$ also hold for this type of maps.

2.3. Non-uniformly expanding local diffeomorphisms. Consider a local diffeomorphism $f: M \rightarrow M$, so that $\mathcal{S}=\emptyset$, which satisfies

- $\left\|(D f)^{-1}\right\| \leq 1$ and

- $K_{1}=\left\{x \in M:\left\|D f(x)^{-1}\right\|=1\right\}$ is finite.

Then by the results in [10] we have that such $f$ has a finite set $\mathbb{E}$ of ergodic equilibrium states for $\phi$ all of which are weak expanding measures. Hence in this case Theorem B holds for every continuous function $\varphi: M \rightarrow \mathbb{R}$.

2.4. Viana maps. The following family of endomorphisms of the cylinder was introduced by Viana in [59]. Let $a_{0} \in(1,2)$ be such that the critical point $x=0$ is preperiodic for the quadratic map $Q(x)=a_{0}-x^{2}$. Let $\mathbb{S}^{1}=\mathbb{R} / \mathbb{Z}$ and $b: \mathbb{S}^{1} \rightarrow \mathbb{R}$ be a Morse function, for instance $b(s)=\sin (2 \pi s)$. For fixed small $\alpha>0$, consider

$$
\begin{array}{rlc}
\hat{f}: \mathbb{S}^{1} \times \mathbb{R} & \longrightarrow & \mathbb{S}^{1} \times \mathbb{R} \\
(s, x) & \longmapsto(\hat{g}(s), \hat{q}(s, x))
\end{array}
$$

where $\hat{g}$ is the uniformly expanding map of the circle defined by $\hat{g}(s)=d \cdot s(\bmod \mathbb{Z})$ for some $d \geq 16$, and $\hat{q}(s, x)=a(s)-x^{2}$ with $a(s)=a_{0}+\alpha b(s)$. For $\alpha>0$ small enough there exists an interval $I \subset(-2,2)$ such that $\hat{f}\left(S^{1} \times I\right)$ is contained in the interior of $S^{1} \times I$. Hence any map $f$ sufficiently $C^{0}$ close to $\hat{f}$ has $S^{1} \times I$ as a forward invariant region. We consider from here on these maps $f$ close to $\hat{f}$ restricted to $\mathbb{S}^{1} \times I$.

In $[59,2,3]$ a $C^{3}$ neighborhood $\mathcal{U}$ of $\hat{f}$ was studied and it was proved that every $f \in \mathcal{U}$ is non-uniformly expanding and has slow recurrence to the non-flat critical set $\mathcal{C}$. The arguments in [59] where extended in [25] to encompass the weaker condition $d \geq 2$ on the expansion of $\hat{g}$, providing the same properties for a $C^{\infty}$-neighborhood $\tilde{\mathcal{U}}$ of $\hat{f}$.

Hence, each $f \subset \mathcal{U}$ or $f \subset \tilde{U}$ is in the seting of Theorem B. Results in [7, 30] show that the tail set decays at least sub-exponentially fast, which is not enough to ensure that Corollaries $\mathrm{C}$ and $\mathrm{E}$ are true for the maps in $\mathcal{U} \cup \tilde{\mathcal{U}}$. It is conjectured that the tail set indeed decays exponentially fast and with a uniform rate for all maps in $\mathcal{U} \cup \tilde{\mathcal{U}}$.

2.5. Partially hyperbolic non-uniformly expanding diffeomorphisms. We sketch the construction of a robust class of partially hyperbolic non-uniformly expanding diffeomorphisms, taking $U$ equal to $M$, following [6]. This construction is closely related to the $C^{1}$ open classes of transitive non-Anosov diffeomorphisms presented in [19, Section 6], as well as other robust examples from [39]. 
Start with a linear Anosov diffeomorphism $\hat{f}$ on the $d$-dimensional torus $M=\mathbb{T}^{d}, d \geq 3$. Write $T M=E \oplus F$ the corresponding hyperbolic decomposition of the tangent bundle with $\operatorname{dim} F \geq 2$. Let $V$ be a small closed domain in $M$ for which there exist unit open cubes $K^{0}$ and $K^{1}$ in $\mathbb{R}^{d}$ such that $V \subset \pi\left(K^{0}\right)$ and $\hat{f}(V) \subset \pi\left(K^{1}\right)$, where $\pi: \mathbb{R}^{d} \rightarrow \mathbb{T}^{d}$ is the canonical projection. Let now $f$ be a diffeomorphism on $\mathbb{T}^{d}$ such that

(A) $f$ admits invariant cone fields $C_{E}$ and $C_{F}$, with small width $a>0$ and containing, respectively, the stable bundle $E$ and the unstable bundle $F$ of $\hat{f}$;

(B) $f$ is partially hyperbolic and volume expanding along the center-unstable direction: there is $\sigma_{1}>1$ so that

$$
\left|\operatorname{det}\left(D f \mid T_{x} \mathcal{D}_{F}\right)\right|>\sigma_{1} \quad \text { and } \quad\left\|D f \mid T_{x} \mathcal{D}_{E}\right\|<\sigma_{1}^{-1}
$$

for any $x \in M$ and any disks $\mathcal{D}_{F}, \mathcal{D}_{E}$ tangent to $C_{F}, C_{E}$, respectively (see Subsection 3.2 for more on invariant cone fields and disks tangent to cone fields in this setting).

(C) $f$ is $C^{1}$-close to $\hat{f}$ in the complement of $V$, so that there exists $\sigma_{2}<1$ satisfying

$$
\left\|\left(D f \mid T_{x} \mathcal{D}_{F}\right)^{-1}\right\|<\sigma_{2} \quad \text { and } \quad\left\|D f \mid T_{x} \mathcal{D}_{E}\right\|<\sigma_{2}
$$

for any $x \in(M \backslash V)$ and any disks $\mathcal{D}_{F}, \mathcal{D}_{E}$ tangent to $C_{F}, C_{E}$, respectively. Moreover $f(V)$ is also contained in the projection of a unit open cube.

(D) there exist some small $\delta_{0}>0$ satisfying

$$
\left\|\left(D f \mid T_{x} \mathcal{D}_{F}\right)^{-1}\right\|<1+\delta_{0}
$$

for any $x \in V$ and any disk $\mathcal{D}_{F}$ tangent to $C_{F}$.

If $\tilde{f}$ is a torus diffeomorphism satisfying (A), (B), (D), and coinciding with $\hat{f}$ outside $V$, then any map $f$ in a $C^{1}$ neighborhood of $\tilde{f}$ satisfies all the previous conditions. Results in $[6$, Appendix] show in particular that for any $f$ satisfying (A)-(D) there exist $c_{u}>0$ such that $f$ is partially hyperbolic and non-uniformly expanding along its center-unstable direction, as defined in Subsection 1.2. Hence on a small $C^{2}$ neighborhood $\mathcal{U}$ of $\tilde{f}$ every diffeomorphism $f \in \mathcal{U}$ satisfies all the conditions of Theorem $\mathrm{D}$ if all equilibrium states with respect to the central-unstable Jacobian have only non-negative Lyapunov exponents along the central-unstable direction. This can be achieved by certain $C^{1}$ perturbations of a linear Anosov diffeomorphism $\hat{f}$.

\section{Hyperbolic times}

The main technical tool used in the study of non-uniformly expanding maps is the notion of hyperbolic times, introduced in $[48,2]$. We say that $n$ is a $(\sigma, \delta, b)$-hyperbolic time of $f$ for a point $x$ if the following two conditions hold with $0<\sigma<1$ and $b, \delta>0$

$$
\prod_{j=n-k}^{n-1}\left\|D f\left(f^{j}(x)\right)^{-1}\right\| \leq \sigma^{k} \quad \text { and } \quad d_{\delta}\left(f^{k}(x), \mathcal{S}\right) \geq e^{-b k}
$$

for all $k=0, \ldots, n-1$.

We now outline the properties of these special times. For detailed proofs see [6, Proposition 2.8] and [3, Proposition 2.6, Corollary 2.7, Proposition 5.2].

Proposition 3.1. There are constants $C_{1}, \delta_{1}>0$ depending on $(\sigma, \delta, b)$ and $f$ only such that, if $n$ is $(\sigma, \delta, b)$-hyperbolic time of $f$ for $x$, then there are hyperbolic preballs $V_{k}(x)$ which are neighborhoods of $f^{n-k}(x), k=1, \ldots, n$, such that 
(1) $f^{k} \mid V_{k}(x)$ maps $V_{k}(x)$ diffeomorphically to the ball of radius $\delta_{1}$ around $f^{n}(x)$;

(2) for every $1 \leq k \leq n$ and $y, z \in V_{k}(x)$

$$
d\left(f^{n-k}(y), f^{n-k}(z)\right) \leq \sigma^{k / 2} \cdot d\left(f^{n}(y), f^{n}(z)\right) ;
$$

(3) for $y, z \in V_{k}(x)$

$$
\frac{1}{C_{1}} \leq \frac{\left|\operatorname{det} D f^{n-k}(y)\right|}{\left|\operatorname{det} D f^{n-k}(z)\right|} \leq C_{1} .
$$

The following ensures existence of infinitely many hyperbolic times for Lebesgue almost every point for non-uniformly expanding maps with slow recurrence to the singular set. A complete proof can be found in [6, Section 5].

Theorem 3.2. Let $f: M \rightarrow M$ be a $C^{1+\alpha}$ local diffeomorphism away from a non-flat singular set $\mathcal{S}$, for some $\alpha \in(0,1)$, non-uniformly expanding and with slow recurrence to $\mathcal{S}$. Then there are $\sigma \in(0,1), \delta, b>0$ and there exists $\theta=\theta(\sigma, \delta, b)>0$ such that Leb-a.e. $x \in M$ has infinitely many $(\sigma, \delta, b)$-hyperbolic times. Moreover if we write $0<n_{1}<n_{2}<n_{2}<\ldots$ for the hyperbolic times of $x$ then their asymptotic frequency satisfies

$$
\liminf _{N \rightarrow \infty} \frac{\#\left\{k \geq 1: n_{k} \leq N\right\}}{N} \geq \theta \quad \text { for } \quad \text { Leb-a.e. } x \in M .
$$

3.1. Coverings by hyperbolic preballs. Here we show how to cover a given measurable subset with hyperbolic preballs, which will enable us to approximate its Lebesgue measure through the measure of families of hyperbolic preballs. In turn, the measure of a hyperbolic preball is related to the Jacobian of the transformations due to bounded distortion.

Lemma 3.3. Let $B \subset M, \theta>0$ and $g: M \rightarrow M$ be a local diffeomorphisms outside $a$ non-flat exceptional set $\mathcal{S}$ such that $g$ has density $>2 \theta$ of hyperbolic times for every $x \in B$. Then, given any probability measure $\nu$ on $B$ and any $m \geq 1$, there exists $n>m$ such that

$$
\nu(\{x \in B: n \text { is a hyperbolic time of } g \text { for } x\})>\frac{\theta}{2} .
$$

This is [44, Lemma 4.4] easily adapted to our setting. For completion we include its very short proof. This lemma shows that we can translate the density of hyperbolic times into the Lebesgue measure of the set of points which have a specific (large) hyperbolic time.

Proof. Let $H$ be the set of pairs $(x, n) \in B \times \mathbb{N}$ for which $n$ is a hyperbolic time of $g$ for $x$. For each $k \geq 1$, let $\#_{k}$ be the normalized counting measure on $\{m+1, m+2, \ldots, m+k\}$. Our assumption implies that for any given $x \in B$ we have for big enough $k \geq 1$

$$
h_{k}(x)=\#_{k}(\pi(H \cap(\{x\} \times \mathbb{N})))>2 \theta,
$$

where $\pi: B \times \mathbb{N} \rightarrow \mathbb{N}$ is the projection on the second coordinate. Given any probability measure $\nu$ on $B$ we have by Fatou's Lemma

$$
\liminf _{k \rightarrow \infty} \int h_{k} d \nu \geq \int \liminf _{k \rightarrow \infty} h_{k} d \nu \geq 2 \theta
$$

so we may fix $k \geq 1$ large enough so that $\nu\left(h_{k}\right)>\theta$ and find a subset for $C \subset B$ with $\nu(C)>1 / 2$ and $h_{k}(x) \geq \theta / 2$ for all $x \in C$. By Fubini's Theorem this means that

$$
\left(\nu \times \#_{k}\right)(H)>\theta \text { and thus } \nu(\hat{\pi}(H \cap(B \times\{n\})))>\frac{\theta}{2}
$$

for some $m<n \leq m+k$, where $\hat{\pi}: B \times \mathbb{N} \rightarrow B$ is the projection on the first coordinate. This proves the lemma. 
3.1.1. Construction of an adequate initial partition. Let $f$ be a regular map in the setting of the Main Theorem with positive density of $(\sigma, \delta)$-hyperbolic times for Lebesgue almost everywhere and $\rho: M \backslash \mathcal{S} \rightarrow(0,+\infty)$ a continuous positive function possibly unbounded. Let $U_{m}=\rho^{-1}\left[e^{-(m+1)}, e^{-m}\right)$ and $N \in \mathbb{Z}^{+}$be such that $U_{m} \neq \emptyset$ and $8 e^{-m}<\delta_{1}$ for $m>N$; and also $U_{N}=M \backslash U_{N+1}$. These sets have non-empty interior and are relatively compact.

Fix $0<\delta_{0}<\delta_{1}$ and let $\mathcal{B}^{N}$ be a finite open cover of $U_{N}$ by $\delta_{0}$-balls and $\mathcal{B}^{m}$ a finite open cover of $U_{m}$ by $r_{m}$-balls, where $r_{m}=\min \left\{e^{-(n+1)}, \delta_{0}\right\} / 8$ for $n>N$. Since $M$ is a finite dimensional manifold, we can find such open cover with a number $\ell_{m}$ of $r_{m}$-balls such that $\ell_{m} \leq C r_{m}^{-\operatorname{dim} M}$ for all sufficiently small $r_{m}$.

Let also $\mathcal{B}=\cup_{m \geq N} \mathcal{B}^{m}$ be a countable open cover of $M \backslash \mathcal{S}$ and let us enumerate the elements of $\mathcal{B}^{N}$ first, then $\mathcal{B}^{N+1}, \mathcal{B}^{N+2}, \ldots$ in this order, obtaining $\mathcal{B}=\left\{B_{k}: k \geq 1\right\}$. From this we define a countable partition $\mathcal{P}$ of $M$ such that $\operatorname{diam} \mathcal{P}(x)<\rho(x)$ following the proof of [39, Lemma 13.3].

We start by setting $P_{1}=B_{1} \cap U_{N}$ as the first element of the partition $\mathcal{P}$. Then, assuming that $P_{1}, \ldots, P_{k}$ are already defined we set $P_{k+1}=B_{k+1} \backslash\left(P_{1} \cup \cdots \cup P_{k}\right)$ for $k+1 \leq \# \mathcal{B}^{N}$. Note that if $P_{k} \neq \emptyset$ then $P_{k}$ has non-empty interior, diameter smaller than $\delta_{0} / 4$ and the boundary $\partial P_{k}$ is a (finite) union of pieces of boundaries of balls in a Riemannian manifold, thus has zero Lebesgue measure. This provides a partition of $U_{N}$ whose nonempty atoms we include in $\mathcal{P}$.

We now repeat this procedure for each $m>N$ obtaining a finite partition of $U_{m}$ whose nonempty atoms we include in $\mathcal{P}$. Note that if $P \in \mathcal{P}$ and $\emptyset \neq P \subset U_{m}$, then $P$ has nonempty interior; $\operatorname{diam} P \leq \min \left\{e^{-(m+1)}, \delta_{0}\right\} / 8 \leq \rho(x), \forall x \in P$ and again $\partial P$ is a finite union of pieces of boundaries of balls in $M$.

Note that since $f$ is regular the boundary of $g(P)$ still has zero Lebesgue measure for every atom $P \in \mathcal{P}$ and every inverse branch $g$ of $f^{n}$, for any $n \geq 1$.

Let us choose one interior point in each atom $P \in \mathcal{P}$ contained in $U_{m}$ and form the set $\mathcal{C}_{m}$ of representatives of the atoms of $\mathcal{P}$ in $U_{m}$; and let $d_{m}=\min \{d(w, \partial \mathcal{P}), w \in$ $\left.\mathcal{C}_{m}\right\}>0$ where $m \geq N$ and $\partial \mathcal{P}=\cup_{P \in \mathcal{P}} \partial P$ is the boundary of $\mathcal{P}$.

Proposition 3.4. Let $\left(\mu_{n}\right)_{n \geq 1}$ be a family of Borel probability measures on $M$; $\mu$ some weak $^{*}$ accumulation point of the sequence $\left(\mu_{n}\right)$ and $\rho: M \backslash \mathcal{S} \rightarrow(0,+\infty)$ be a continuous $\mu$-integrable function. Then, given $0<\xi \leq \tau$, there exists a partition $\mathcal{P}_{\xi, \tau}$ with the same number of atoms of $\mathcal{P}$ in each $U_{m}, m \geq N$, each atom has non-empty interior and zero Lebesgue measure boundary; and also

(1) $\mu\left(\partial \mathcal{P}_{\xi, \tau}\right)=0$ and $\mu_{n}\left(\partial \mathcal{P}_{\xi, \tau}\right)=0$ for all $n \geq 1$;

(2) each $P \in \mathcal{P}_{\xi, \tau}$ contains one, and only one, element of $\mathcal{C}=\sum_{m \geq N} \mathcal{C}_{m}{ }^{1}$;

(3) $2 \operatorname{diam} \mathcal{P}_{\xi, \tau}(x) \leq \min \left\{\rho(x), \tau_{1}\right\}$ for Leb-, $\mu$ - and $\mu_{n}$-a.e. $x$;

(4) for each $P \in \mathcal{P}_{\xi, \tau}$ there is $Q \in \mathcal{P}$ satisfying $\operatorname{Leb}(P \triangle Q)<\xi<\tau \cdot \operatorname{Leb}(Q)$;

(5) $H_{\mu}\left(\mathcal{P}_{\xi, \tau}\right)<\infty$.

Proof. Let $0<\xi<\tau>0$ be given. For each fixed $m \geq N$, let us take $0<\gamma_{m}<$ $\min \left\{\xi, d_{m}, r_{m}^{3}\right\}$ such that for any given $r_{m}$-ball $B=B\left(x, r_{m}\right) \in \mathcal{B}^{m}$

$$
\operatorname{Leb}\left(B\left(x, r_{m}+\gamma_{m}\right) \backslash B\left(x, r_{m}\right)\right)<\varepsilon_{m} / \# \mathcal{B}^{m}
$$

\footnotetext{
${ }^{1}$ We write $A+B$ the union of the disjoint subsets $A$ and $B$.
} 
where $0<\varepsilon_{m}<\min \left\{\xi, \tau \cdot \min \left\{\operatorname{Leb}(B): B \in \mathcal{P}, B \subset U_{m}\right\}\right\}$; and also for all $n \geq 1$

$$
\mu\left(\partial B\left(x, r_{m}+\gamma_{m}\right)\right)=0=\mu_{n}\left(\partial B\left(x, r_{m}+\gamma_{m}\right)\right)
$$

and in addition for $a_{m}=\left(1-\gamma_{m}\right) e^{-m}$

$$
\operatorname{Leb}\left(\rho^{-1}\left(a_{m}\right)\right)=0=\mu\left(\rho^{-1}\left(a_{m}\right)\right)=\mu_{n}\left(\rho^{-1}\left(a_{m}\right)\right) .
$$

Such value of $\gamma_{m}$ exists since the set of such values so that some of the expressions in (3.3) or (3.4) is positive for some $B \in \mathcal{B}^{m}$, some $m \geq N$ and some $n \geq 1$ is denumerable. Thus we may take $\gamma_{m}>0$ satisfying (3.3) and (3.4) arbitrarily close to zero, and so inequality (3.2) can also be obtained.

We consider now the open cover $\widetilde{\mathcal{B}}$ of $M \backslash \mathcal{S}$ obtained by replacing $U_{m}$ by $\widetilde{U_{m}}=$ $\rho^{-1}\left[a_{m+1}, a_{m}\right)$ and each $r_{m}$-ball of $\mathcal{B}^{m}$ by a concentric $\left(r_{m}+\gamma_{m}\right)$-ball in $\widetilde{\mathcal{B}^{m}}$ for each $m \geq N$, and construct the partition $\widetilde{\mathcal{P}}$ obtained from $\widetilde{\mathcal{B}}=\sum_{m \geq N} \widehat{\mathcal{B}^{m}}$ by the same procedure as before with the same order. Since $\gamma_{m}<\varepsilon<\bar{d}_{m}$ we obtain $d\left(w, \partial \mathcal{P}_{\varepsilon}\right) \geq d_{m}-\gamma_{m}>0$ for all $w \in \mathcal{C}_{m}, m \geq N$.

This shows that each $w \in \mathcal{C}$ is contained in some atom $P_{w}$ of $\widetilde{\mathcal{P}}$. Moreover there cannot be distinct $w_{1}, w_{2} \in \mathcal{C}$ such that $w_{2} \in P_{w_{1}}$, because this would mean that for some $m \geq N$ and $B=B\left(x, r_{m}\right) \in \mathcal{B}^{m}$ we have $w_{2} \in B\left(x, r_{m}\right), w_{1} \notin B\left(x, r_{m}\right)$ and $w_{1}, w_{2} \in B\left(x, r_{m}+\gamma_{m}\right)$, which contradicts the choice of $\gamma_{m}<d_{m}$.

Hence, on the one hand, $\# \mathcal{P} \leq \# \widetilde{\mathcal{P}}$. On the other hand, let us consider $\left\{P_{w}, w \in \mathcal{C}\right\}$. There might be other (finitely many) atoms $P$ in $\widetilde{\mathcal{P}}$ and, if so, we join them to some adjacent atom $P_{w}$ (meaning $\bar{P} \cap \bar{P}_{w} \neq \emptyset$ ) obtaining a new atom $P \cup P_{w}$. In this way we obtain a partition, which we still denote by $\widetilde{\mathcal{P}}$ with as many atoms as the elements of $\mathcal{C}$ and satisfying items (1) and (2) of the statement of the lemma.

Finally, for any $w \in \mathcal{C}$ the corresponding atoms $P_{w} \in \widetilde{\mathcal{P}}$ and $Q_{w} \in \mathcal{P}$ satisfy $P_{w} \in \widetilde{\mathcal{B}^{m}}, Q_{w} \in \mathcal{B}^{m}$ for some $m \geq N$ and

$$
\operatorname{Leb}\left(P_{w} \triangle Q_{w}\right) \leq \sum_{B\left(x, r_{m}\right) \in \mathcal{B}^{m}} \operatorname{Leb}\left(B\left(x, r_{m}+\gamma_{m}\right) \backslash B\left(x, r_{m}\right)\right)<\# \mathcal{B}^{m} \cdot \varepsilon_{m} / \# \mathcal{B}^{m}=\varepsilon_{m} \leq \varepsilon
$$

and $\operatorname{diam}\left(P_{w}\right) \leq 4 r_{m}<\min \left\{\rho(x), \delta_{1}\right\} / 2$ for all $x \in P_{w}$. This provides item (3) of the statement of the lemma. By the choice of $\varepsilon_{m}$ we also get

$$
\operatorname{Leb}\left(P_{w} \triangle Q_{w}\right)<\varepsilon_{m} \leq \tau \cdot \min \left\{\operatorname{Leb}(B): B \in \mathcal{P}, B \subset U_{m}\right\} \leq \tau \cdot \operatorname{Leb}\left(Q_{w}\right) .
$$

This is item (4) of the statement of the lemma. To prove that $\widetilde{\mathcal{P}}$ has finite entropy, we use observe that the number of atoms of $\widetilde{\mathcal{P}}$ on each $U_{m}$ is bounded by $\ell_{m}$, and so by construction we obtain

$$
\begin{aligned}
H_{\mu}(\widetilde{\mathcal{P}}) & =\sum_{m \geq N} \sum_{P \in \widetilde{\mathcal{P}}: P \subset U_{m}}-\mu(P) \log \mu(P)=\sum_{m \geq N} \sum_{P \in \widetilde{\mathcal{P}}: P \subset U_{m}} \mu\left(U_{n}\right)\left(-\frac{\mu(P)}{\mu\left(U_{n}\right)} \log \frac{\mu(P)}{\mu\left(U_{n}\right)}-\log \mu\left(U_{n}\right)\right) \\
& \leq \sum_{m \geq N} \mu\left(U_{n}\right)\left(\log \ell_{m}-\log \mu\left(U_{n}\right)\right) \\
& \leq \sum_{m \geq N}\left(-\mu\left(U_{n}\right) \log \mu\left(U_{n}\right)+\mu\left(U_{n}\right) \log C+\operatorname{dim}(M)(m+1) \mu\left(U_{m}\right)\right)
\end{aligned}
$$


Then we deduce the summability of the above series using that $\log \rho \in L^{1}(\mu)$, as follows. On the one hand, we note that

$$
\sum_{m \geq N}(m+1) \mu\left(U_{m}\right)=1+\sum_{m \geq N}-\log e^{-m} \mu\left(U_{m}\right) \leq 1+\int|\log \rho| d \mu<\infty .
$$

\section{On the other hand, we have}

Lemma 3.5 (Lemma 13.2 from [39]). If $x_{n} \in(0,1), n \geq 1$ and $\sum_{n \geq 1} n x_{n}<\infty$, then $\sum_{n \geq 1} x_{n} \log \left(1 / x_{n}\right)<\infty$.

Now setting $x_{n}=\mu\left(U_{n}\right)$ we have the assumption of Lemma 3.5 from (3.7) and so we deduce

$$
\sum_{n \geq N}-\mu\left(U_{n}\right) \log \mu\left(U_{n}\right)=\sum_{n \geq N} x_{n} \log \left(1 / x_{n}\right)<\infty .
$$

This completes the proof of the summability of (3.6) and with it the proof of Proposition 3.4 after setting $\mathcal{P}_{\xi, \tau}=\widetilde{\mathcal{P}}$.

3.1.2. The flexible covering lemma. Having this we can now obtain the following flexible covering lemma.

Lemma 3.6. Let a measurable set $E \subset M, m \geq 1$ and $\varepsilon>0$ be given with $\operatorname{Leb}(E)>0$. Let $\theta>0$ be a lower bound for the density of hyperbolic times for Lebesgue almost every point. Then there are integers $m<n_{1}<\cdots<n_{k}$ for $k=k(\varepsilon) \geq 1$ and families $\mathcal{E}_{i}$ of subsets of $M$, $i=1, \ldots, k$ such that

(1) $\mathcal{E}_{1} \cup \cdots \cup \mathcal{E}_{k}$ is a finite pairwise disjoint family of subsets of $M$;

(2) $n_{i}$ is a $(\sigma / 2, \delta / 2)$-hyperbolic time for every point in $P$, for every element $P \in \mathcal{E}_{i}$, $i=1, \ldots, k$;

(3) every $P \in \mathcal{E}_{i}$ is the preimage of some element $Q \in \mathcal{P}$ under an inverse branch of $f^{n_{i}}$, $i=1, \ldots, k$;

(4) there is an open set $U_{1} \supset E$ containing the elements of $\mathcal{E}_{1} \cup \cdots \cup \mathcal{E}_{k}$ with $\operatorname{Leb}\left(U_{1} \backslash E\right)<$ $\varepsilon$;

(5) $\operatorname{Leb}\left(E \triangle \bigcup_{i} \varepsilon_{i}\right) \leq\left(1-\frac{\theta}{4}\right)^{k}<\varepsilon$.

The proof follows [44, Lemma 8.2] closely. We write $\mathcal{C}_{m}$ the set of pairs $\left(z, n_{i}\right)$ where $f^{n_{i}}(z)=w \in \mathcal{C}$ and $z \in P$ for all $P \in \mathcal{E}_{i}$ and $i=1, \ldots, k$ (such $z$ exist by item (3) of Lemma 3.6).

Remark 3.7. Note that $k$ depends on $\varepsilon$ only and not on the set $E$.

Proof of Lemma 3.6. By the non-uniformly expanding assumption on $f$ we know that there exists $\theta>0$ such that Lebesgue almost every point has density $>\theta$ of hyperbolic times of $f$.

Let $U_{1}$ be an open set and $K_{1}$ a compact set such that $K_{1} \subset E \subset U_{1}$ and $\operatorname{Leb}\left(U_{1} \backslash K_{1}\right)<\varepsilon$ and $\operatorname{Leb}\left(K_{1}\right)>(1 / 2) \operatorname{Leb}\left(U_{1}\right)$. Using Lemma 3.3 with $B=K_{1}$ and $\nu=\operatorname{Leb} / \operatorname{Leb}\left(K_{1}\right)$ we can find $n_{1}>m$ such that $e^{-c n_{1}}<d\left(K_{1}, M \backslash U_{1}\right)$ and the subset $L_{1}$ of points of $K_{1}$ for which $n_{1}$ is a hyperbolic time satisfies $\operatorname{Leb}\left(L_{1}\right) \geq \frac{\theta}{2} \operatorname{Leb}\left(K_{1}\right) \geq \frac{\theta}{4} \operatorname{Leb}(E)$.

Given $x \in L_{1}$ let $g: B\left(f^{n_{1}}(x), \delta_{1}\right) \rightarrow V_{n_{1}}(x)$ be the inverse branch of $f^{n_{1}} \mid V_{n_{1}}(x)$, recall that $n_{1}$ is a hyperbolic time for $x$ and see Proposition 3.1. By the choice of $\mathcal{P}$ from Proposition 3.4 there exists a unique $P \in \mathcal{P}$ such that $f^{n_{1}}(x) \in P$. Let us consider $g(P)$ 
and let $\mathcal{E}_{1}$ be the family of all such sets obtained as $g(P)$ which intersect $L_{1}$, where $g$ is an inverse branch of $f^{n_{1}}$ corresponding to a hyperbolic time and $P$ is an element of $\mathcal{P}$.

Note that the elements of $\varepsilon_{1}$ are pairwise disjoint because $\mathcal{P}$ is a partition. Moreover by the properties of hyperbolic times (Proposition 3.1) the diameter of $P \in \mathcal{E}_{1}$ is smaller than $e^{-c n_{1}}$. Hence the union $E_{1}$ of all the elements of $\mathcal{E}_{1}$ is contained in $U_{1}$ and by construction

$$
\operatorname{Leb}\left(E_{1} \cap E\right) \geq \operatorname{Leb}\left(L_{1}\right) \geq \frac{\theta}{4} \operatorname{Leb}(E) .
$$

Now consider the open set $U_{2}=U_{1} \backslash \overline{E_{1}}$ and set $K_{2} \subset E \backslash \overline{E_{1}}$ a compact set such that $\operatorname{Leb}\left(K_{2}\right) \geq(1 / 2) \operatorname{Leb}\left(E \backslash E_{1}\right)$. Observe that $\operatorname{Leb}\left(\overline{E_{1}} \backslash E_{1}\right)=0$ since $\partial \mathcal{P}$ has zero Lebesgue measure and this property is preserved under backward iteration by the regularity assumption on $f$. Reasoning as before, we can find $n_{2}>n_{1}$ such that $e^{-c n_{2}}<d\left(K_{2}, M \backslash U_{2}\right)$ and a set $L_{2} \subset K_{2}$ such that $\operatorname{Leb}\left(L_{2}\right) \geq\left(\frac{\theta}{2}\right) \operatorname{Leb}\left(K_{2}\right)$ and $n_{2}$ is a hyperbolic time for every $x \in L_{2}$. Let $\mathcal{E}_{2}$ be the family of elements $g(P)$ which intersect $L_{2}$, where $P \in \mathcal{P}$ and $g$ is an inverse branch of $f^{n_{1}}$ corresponding to a hyperbolic time.

Again $\mathcal{E}_{2}$ is a pairwise disjoint family of sets whose diameters are smaller than $e^{-c n_{2}}$. Thus their union $E_{2}$ is contained in $U_{2}$. Hence $\mathcal{E}_{1} \cup \mathcal{E}_{2}$ is also a pairwise disjoint family and, in addition

$$
\operatorname{Leb}\left(E_{2} \cap\left(E \backslash E_{1}\right)\right) \geq \operatorname{Leb}\left(L_{2}\right) \geq \frac{\theta}{2} \operatorname{Leb}\left(K_{2}\right) \geq \frac{\theta}{4} \operatorname{Leb}\left(E \backslash E_{1}\right) .
$$

Repeating this procedure we obtain families $\mathcal{E}_{i}, i=1, \ldots, k$ of elements of $\mathcal{P}_{n_{i}}$ which are pairwise disjoint and contained in $U_{1}$, and

$$
\operatorname{Leb}\left(E_{i+1} \cap\left(E \backslash\left(E_{1} \cup \cdots \cup E_{i}\right)\right)\right) \geq \frac{\theta}{4} \operatorname{Leb}\left(E \backslash\left(E_{1} \cup \cdots \cup E_{i}\right)\right)
$$

for all $i=1, \ldots, k-1$, for some $k \geq 1$, where $E_{j}=\cup \mathcal{E}_{j}$. Hence

$$
\operatorname{Leb}\left(\bigcup_{i=1}^{k} E_{i} \backslash E\right) \leq \operatorname{Leb}\left(U_{1} \backslash E\right)<\varepsilon
$$

and (3.8) ensures that

$$
\operatorname{Leb}\left(E \backslash \bigcup_{i=1}^{k} E_{i}\right) \leq\left(1-\frac{\theta}{4}\right)^{k} \operatorname{Leb}(E) .
$$

Therefore we can find $k \geq 1$ such that Leb $\left(E \triangle \cup_{i=1}^{k} \varepsilon_{i}\right)<\varepsilon$, as stated.

Remark 3.8. Note that the construction proving Lemma 3.6 gives a finite sequence of hyperbolic times, open sets $U_{1}, \ldots, U_{k}$ and closed sets $\bar{E}_{1}, \ldots, \bar{E}_{k}$. Having these we can find small enough $\delta>\varepsilon>0$, replace $\mathcal{P}$ in the proof of Lemma 3.6 by any partition $\mathcal{P}_{\varepsilon, \delta}$ obtained as in Proposition 3.4 (by slightly modifying $\mathcal{P}$ ), and use the same inverse branches to obtain families $\mathcal{E}_{i}^{\prime}$ of preballs such that

$$
\operatorname{Leb}\left(\left(\bigcup_{i} \mathcal{E}_{i}\right) \triangle\left(\bigcup_{i} \mathcal{E}_{i}^{\prime}\right)\right) \leq \sum_{i} C_{1} \delta \operatorname{Leb}\left(\varepsilon_{i}\right)<C_{1} \delta \operatorname{Leb}\left(\bigcup_{i} \mathcal{E}_{i}\right) \leq C_{1} \delta
$$

where $C_{1}$ is the volume distortion constant (see Proposition 3.1). Hence, after the modification of the initial partition, we get

$$
\operatorname{Leb}\left(E \triangle \bigcup_{i} \mathcal{E}_{i}^{\prime}\right)<\varepsilon+C_{1} \delta<\left(1+C_{1}\right) \delta
$$


since $\varepsilon<\delta$. Moreover the set $\mathcal{C}_{m}$ is unaffected since $\mathcal{C}$ is fixed and the inverse branches are kept.

3.2. The partially hyperbolic setting. Here we state the main results needed to obtain an extension of the covering Lemma 3.6 to the setting of partially hyperbolic non-uniformly expanding attracting sets. As we indicate along the way, the proofs of most of them can be found in [6].

3.2.1. Stable/Unstable cone fields. Let $\Lambda$ be a partially hyperbolic and non-uniformly expanding attracting set for a $C^{2}$ diffeomorphism $f: M \rightarrow M$ with a trapping region $U \subset M$. The existence of the dominated splitting $E \oplus F$ of $T_{\Lambda} M$ ensures the existence of a continuous extension $\tilde{E} \oplus \tilde{F}$ of $E \oplus F$ to a neighborhood of $\Lambda$, which we assume without loss to be $U$, and of the following cone fields:

stable cones: $\mathbb{E}_{x}^{a}=\{(u, v) \in \tilde{E}(x) \oplus \tilde{F}(x):\|v\| \leq a \cdot\|u\|\}$;

unstable cones: $\mathbb{F}_{x}^{b}=\{(u, v) \in \tilde{E}(x) \oplus \tilde{F}(x):\|u\| \leq b \cdot\|v\|\}$;

for all $x \in U$ and $a, b \in(0,1)$, which are $D f$-invariant in the following sense (see e.g. [18, Appendix C])

- if $x, f^{-1}(x) \in U$, then $D f^{-1}\left(\mathbb{E}_{x}^{a}\right) \subset \mathbb{E}_{f^{-1}(x)}^{\lambda a}$;

- if $x, f(x) \in U$, then $D f\left(\mathbb{F}_{x}^{b}\right) \subset \mathbb{F}_{f(x)}^{\lambda b}$;

for some $\lambda \in(0,1)$. Continuity enables us to unambiguously denote $d_{E}=\operatorname{dim}(\tilde{E})$ and $d_{F}=\operatorname{dim}(\tilde{F})$, so that $d=d_{E}+d_{F}=\operatorname{dim}(M)$, and domination guarantees that the angles between the $\tilde{E}$ and $\tilde{F}$ directions are bounded from below away from zero at every point.

3.2.2. Hyperbolic times. In this setting, given $\sigma>1$ we say that $n$ is a $\sigma$-hyperbolic time for $x \in U$ if

$$
\prod_{j=n-k+1}^{n}\left\|\left(D f \mid F_{f^{j}(x)}\right)^{-1}\right\| \leq \sigma^{k} \quad \text { for all } 1 \leq k \leq n .
$$

Remark 3.9. This definition of hyperbolic time is entirely analogous to the one given in the local diffeomorphisms setting except that we restrict the derivatives to the $F$-direction. Hence the statement and proof of Lemma 3.3 carry over without change.

3.2.3. E-disks and $F$-disks. Let us fix the unit balls of dimensions $d_{E}, d_{F}$

$$
\mathbb{B}_{E}=\left\{w \in \mathbb{R}^{d_{E}}:\|w\|_{2} \leq 1\right\} \quad \text { and } \quad \mathbb{B}_{F}=\left\{w \in \mathbb{R}^{d_{F}}:\|w\|_{2} \leq 1\right\}
$$

where $\|\cdot\|_{2}$ is the standard Euclidean norm on the corresponding Euclidean space. We say that a $C^{1+\alpha}$ embedding $\Delta: \mathbb{B}_{E} \rightarrow M$ (respectively $\Delta: \mathbb{B}_{F} \rightarrow M$ ) is a $E$-disk (resp. $F$-disk) if the image of $D \Delta(w)$ is contained in $\mathbb{E}_{\Delta(w)}^{a}$ for all $w \in \mathbb{B}_{E}\left(\right.$ resp. $D \Delta(w)\left(\mathbb{R}^{d_{F}}\right) \subset \mathbb{F}_{\Delta(w)}^{b}$ for every $\left.w \in \mathbb{B}_{F}\right)$, where $\alpha \in(0,1)$ if fixed.

3.2.4. Curvature of $E$ - and $F$-disks at hyperbolic times. Let $r_{0}>0$ be an injectivity radius of the exponential map on $M$, that is $\exp _{x}: B\left(x, r_{0}\right) \rightarrow M$ is a diffeomorphism onto its image $G\left(x, r_{0}\right)=\exp _{x}\left(B\left(x, r_{0}\right)\right)$, where $B\left(x, r_{0}\right)=\left\{v \in T_{X} M:\|v\|<r_{0}\right\}$ is the $r_{0}$-neighborhood of 0 in $T_{x} M$. By the continuity of the splitting $E \oplus F$ and the cone fields we can choose $0<r<\min \left\{r_{0}, \delta_{1} / 4\right\}$ such that for every $x \in \Lambda$ the subspace $E_{x}$ is contained in all the images 
of the cone field $\mathbb{E}_{x}^{a}$ under the exponential map $\exp _{x}$ and analogously for the complementary direction, that is for every $y \in G(x, r) \cap \Lambda$ we have

$$
E_{x} \subset D\left(\exp _{x}^{-1}\right)\left(\mathbb{E}_{y}^{a}\right) \quad \text { and } \quad F_{x} \subset D\left(\exp _{x}^{-1}\right)\left(\mathbb{F}_{y}^{b}\right)
$$

This ensures that every $F$-disk (respectively every $E$-disk) $\Delta$ is such that its image on $B(x, r)$ given by $\exp _{x}^{-1}(\Delta \cap G(x, r))$ is transversal to the direction of $E_{x}$ (resp. $F_{x}$ ).

The "curvature" of $E$ - and $F$-disks can be determined by the notion of Hölder variation of the tangent bundle as follows.

We write $\Delta$ also for the image of the respective embedding for every $E$ - or $F$-disk. Hence if $\Delta$ is a $E$-disk and $y=\Delta(w)$ for some $w \in \mathbb{B}_{E}$, then the tangent space of $\Delta$ at $y$ is the graph of a linear map $A_{x}(y): T_{x} \Delta \rightarrow F(x)$ for $w \in \Delta^{-1}\left(V_{x}\right)$ (here $T_{x} \Delta=D \Delta(x)\left(\mathbb{R}^{d_{E}}\right)$ ). The same happens locally for a $F$-disk exchanging the roles of the bundles $E$ and $F$ above.

The domination condition on the splitting $E \oplus F$ ensures the existence of $\zeta \in(0,1)$ such that for some $n \geq 1$ and all $x \in \Lambda$

$$
\left\|D f^{n} \mid E_{x}\right\| \cdot\left\|\left(D f^{n} \mid F_{x}\right)^{-1}\right\|^{1+\zeta} \leq \frac{3}{4}
$$

Given $C>0$ we say that the tangent bundle of $\Delta$ is $(C, \zeta)$-Hölder if

$$
\left\|A_{x}(y)\right\| \leq C \operatorname{dist}_{\Delta}(x, y)^{\zeta} \quad \text { for all } y \in G(x, r) \cap \Delta \quad \text { and } \quad x \in U,
$$

where $\operatorname{dist}_{\Delta}(x, y)$ is the distance along $\Delta$ defined by the length of the shortest smooth curve from $x$ to $y$ inside $\Delta$ calculated with respect to the Riemannian norm $\|\cdot\|$ induced on $T M$.

For a $E$ - or $F$-disk $\Delta \subset U$ we define

$$
\kappa(\Delta)=\inf \{C>0: T \Delta \text { is }(C, \zeta) \text {-Hölder }\} .
$$

The proof of the following result can be found in [6, Subsection 2.1]. The basic ingredients are the cone invariance and dominated decomposition properties for $f$.

Proposition 3.10. There is $C_{2}>0$ such that given a F-disk $\Delta \subset U$

(1) there exists $n_{1} \in \mathbb{N}$ such that $\kappa\left(f^{n}(\Delta)\right) \leq C_{2}$ for all $n \geq n_{1}$;

(2) if $\kappa(\Delta) \leq C_{2}$ then $\kappa\left(f^{n}(\Delta)\right) \leq C_{2}$ for all $n \geq 0$;

(3) in particular, if $\Delta$ is as in the previous item, then

$$
J_{n}: f^{n}(\Delta) \ni x \mapsto \log \mid \operatorname{det}\left(D f\left|T_{x}\left(f^{n}(\Delta)\right)\right|\right.
$$

is $\left(L_{1}, \zeta\right)$-Hölder continuous with $L_{1}>0$ depending only on $C_{2}$ and $f$, for every $n \geq 1$.

3.2.5. Distortion bounds. The following uniform backward contraction and distortion bounds are proved in [6, Lemma 2.7, Proposition 2.8].

Proposition 3.11. There exist $C_{3}, \delta_{1}>0$ depending only on $f, \sigma$ such that, given any $F$-disk $\Delta \subset U, x \in \Delta$, and $n \geq 1$ a $\sigma$-hyperbolic time for $x$,

(1) $\operatorname{dist}_{f^{n-k}(D)}\left(f^{n-k}(y), f^{n-k}(x)\right) \leq \sigma^{k / 2} \operatorname{dist}_{f^{n}(D)}\left(f^{n}(y), f^{n}(x)\right)$, for all $y \in \Delta$ satisfying $\operatorname{dist}\left(f^{n}(x), f^{n}(y)\right) \leq \delta_{1}$;

(2) if $\kappa(\Delta) \leq C_{2}$ then

$$
\frac{1}{C_{3}} \leq \frac{\left|\operatorname{det} D f^{n}\right| T_{y} \Delta \mid}{\left|\operatorname{det} D f^{n}\right| T_{x} \Delta \mid} \leq C_{3}
$$

for every $y \in \Delta$ such that $\operatorname{dist}\left(f^{n}(y), f^{n}(x)\right) \leq \delta_{1}$. 
3.2.6. The initial partition and the covering lemma. Now we consider the following rectangle

$$
\hat{R}(x, s)=\left\{(u, v) \in T_{x} M:\|u\|<s,\|v\|<s, u \in E_{x}, v \in F_{x}\right\}
$$

where $s$ is chosen so that $\hat{R}(x, s) \subset B_{x}(r)$ for all $x \in \Lambda$. This defines an open cover $\left\{\exp _{x}(\hat{R}(x, s))\right\}_{x \in \Lambda}$ of $\Lambda$ which admits a finite subcover denoted by $\mathcal{R}=\left\{R_{1}=R\left(x_{1}, s\right), \ldots, R_{h}=\right.$ $\left.R\left(x_{h}, s\right)\right\}$. This finite cover will define the initial partition $\mathcal{P}$ given by

$$
\mathcal{P}=\left\{R_{1}, M \backslash R_{1}\right\} \vee \cdots \vee\left\{R_{h}, M \backslash R_{h}\right\} .
$$

We may assume without loss that $\operatorname{Leb}(\partial \mathcal{P})=0$ by slightly changing the initial cover. We choose an interior point in each element of $\mathcal{P}$ which together define the set $\mathcal{C}$.

Now we adapt the covering Lemma 3.6 to the setting of partially hyperbolic non-uniformly expanding attracting sets as follows.

Lemma 3.12. Let a measurable set $E \subset U, m \geq 1$ and $\varepsilon>0$ be given. Let $\theta>0$ be a lower bound for the density of hyperbolic times for Lebesgue almost every point on $U$. Then there are integers $m<n_{1}<\cdots<n_{k}$ for $k=k(\varepsilon) \geq 1$, and families $\mathcal{E}_{i}$ of subsets of $M, i=1, \ldots, k$ such that

(1) $\mathcal{E}_{1} \cup \cdots \cup \mathcal{E}_{k}$ is a finite family of subsets of $M$ and each $\mathcal{E}_{i}$ is a pairwise disjoint family;

(2) $n_{i}$ is a $(\sigma / 2, \delta / 2)$-hyperbolic time for every point in $P$, for every element $P \in \mathcal{E}_{i}$, $i=1, \ldots, k$;

(3) every $P \in \mathcal{E}_{i}$ is the preimage of some element $Q \in \mathcal{P}$ under $f^{-n_{i}}, i=1, \ldots, k$;

(4) $\operatorname{Leb}\left(E \backslash \bigcup_{i} \varepsilon_{i}\right) \leq\left(1-\frac{\theta}{4}\right)^{k}<\varepsilon$.

Proof. Let $E \subset U, \varepsilon>0$ and $m \geq 1$ be given. Set $\nu=\operatorname{Leb} / \operatorname{Leb}(E)$ and apply Lemma 3.3 with $B=E$ to obtain $n_{1}>m$ and $L_{1} \subset E$ such that $n_{1}$ is a hyperbolic time for every point $x \in L_{1}$ and $\operatorname{Leb}\left(L_{1}\right) \geq \frac{\theta}{2} \operatorname{Leb}(E)$.

Given $x \in L_{1}$ let $P_{x}$ be the unique element of the partition $f^{-n_{1}} \mathcal{P}$ which contains $x$ (recall that $f$ is a diffeomorphism). Define $\mathcal{E}_{1}=\left\{P_{x}: x \in L_{1}\right\}$. Then $\mathcal{E}_{1}$ is a finite pairwise disjoint family of preimages of elements of $\mathcal{P}$ corresponding to a hyperbolic time $n_{1}$. If $E_{1}$ is the union of the elements of $\varepsilon_{1}$, then

$$
\operatorname{Leb}\left(E_{1} \cap E\right) \geq \operatorname{Leb}\left(L_{1}\right) \geq \frac{\theta}{2} \operatorname{Leb}(E) .
$$

Now consider $\hat{E}_{2}=E \backslash \bar{E}_{1}$. If $\operatorname{Leb}\left(\hat{E}_{2}\right)<\varepsilon$ then we are done, since then $\operatorname{Leb}\left(E \backslash E_{1}\right)<\varepsilon$ because $\operatorname{Leb}\left(\partial \mathcal{E}_{1}\right)=0$ as $f$ is regular map. Otherwise use again Lemma 3.3 to find $n_{2}>n_{1}$ and $L_{2} \subset \hat{E}_{2}$ such that $n_{2}$ is a hyperbolic time for all points of $L_{2}$ and $\operatorname{Leb}\left(L_{2}\right) \geq \frac{\theta}{2} \operatorname{Leb}\left(\hat{E}_{2}\right)$.

Let $\mathcal{E}_{2}$ be the family of all elements of the partition $f^{-n_{2}} \mathcal{P}$ which intersect $\hat{E}_{2}$. Then $\mathcal{E}_{2}$ is a pairwise disjoint family and the union $E_{2}$ of its elements satisfies

$$
\operatorname{Leb}\left(E_{2} \cap\left(E \backslash E_{1}\right)\right) \geq \operatorname{Leb}\left(L_{2}\right) \geq \frac{\theta}{2} \operatorname{Leb}\left(\hat{E}_{2}\right) \geq \frac{\theta}{4} \operatorname{Leb}\left(E \backslash E_{1}\right) .
$$

Repeating this procedure we get families $\mathcal{E}_{i}, i=1, \ldots, k$ of elements of $f^{-n_{i}} \mathcal{P}$ with $m<n_{1}<$ $\cdots<n_{k}$ satisfying the inequality (3.8). These families satisfy items (1)-(3) by construction and item (4) follows by (3.8) as in the proof of Lemma 3.6. This concludes the proof.

Observe that we may apply Proposition 3.4 to $\mathcal{P}$ with $\rho=$ const. to ensure that, for a given denumerable family of $f$-invariant probability measures, there is a partition $\mathcal{P}_{\xi, \tau}$ arbitrarily close to $\mathcal{P}$, with the same number of elements, such that the measure of the 
boundary of the elements of $\mathcal{P}_{\xi, \tau}$ is zero with respect to all measures of the family. Moreover, as in the previous subsection, we write $\mathcal{C}_{m}$ the set of pairs $\left(z, n_{i}\right)$ where $f^{n_{i}}(z)=w \in \mathcal{C}$ and $z \in P$ for all $P \in \mathcal{E}_{i}$ and $i=1, \ldots, k$. In addition, we can build the new partition $\mathcal{P}_{\xi, \tau}$ in such a way that the sets $\mathcal{C}_{n}$ are unchanged.

3.3. The volume of dynamical balls. Here we show that the volume of dynamical balls on hyperbolic times is well controlled by $S_{n} J$, either in the local diffeomorphism case with or without singularities, or in the partially hyperbolic case.

3.3.1. The local diffeomorphism case with singularities. Note that by the properties of bounded distortion of volumes during hyperbolic times (item 3 of Proposition 3.1) we can write, if $n$ is a hyperbolic time of $f$ for $x \in M$

$$
\begin{aligned}
\operatorname{Leb}\left(B\left(f^{k}(x), n-k, \delta_{1}\right)\right) & =\int_{B\left(f^{k}(x), n-k, \delta_{1}\right)} \frac{d z}{\left|\operatorname{det} D f^{n-k}(z)\right|} \\
& \leq C_{1} \frac{\operatorname{Leb}\left(B\left(f^{n}(x), \delta_{1}\right)\right)}{\left|\operatorname{det} D f^{n-k}(x)\right|},
\end{aligned}
$$

then recalling that $J=\log |\operatorname{det} D f|$ we get

$$
\begin{aligned}
\operatorname{Leb}\left(B\left(f^{k}(x), n-k, \delta_{1}\right)\right) & \leq C_{1} e^{-S_{n-k} J\left(f^{k}(x)\right)} \operatorname{Leb}\left(B\left(f^{n}(x), \delta_{1}\right)\right. \\
& \leq C_{1} e^{-S_{n-k} J\left(f^{k}(x)\right)} .
\end{aligned}
$$

Observe that by Proposition 3.1 if $n$ is a hyperbolic time of $f$ for $x$ we get due to uniform backward contraction

$$
S_{n-k} J\left(f^{k}(x)\right)=\log \left|\operatorname{det} D f^{n-k}(x)\right| \geq(n-k) \cdot \operatorname{dim}(M) \log \sigma / 2>0
$$

which will be used several times in what follows.

3.3.2. The partially hyperbolic case with non-uniform expansion. In the partially hyperbolic and non-uniformly expanding setting we recall the construction of the cover $\mathcal{R}=\left\{R_{1}, \ldots, R_{j}\right\}$ and the initial partition $\mathcal{P}$ from Subsection 3.2. Observe that if we take $\delta_{0}$ to be the Lebesgue number of the covering $\mathcal{R}$ (see e.g. [43]), then for all $0<\delta<\delta_{0}$ we have for all $x \in U$ and $n \geq 1$ a hyperbolic time for $x$

$$
B(x, n, \delta) \subset f^{-n \mathcal{P}}(x),
$$

where $f^{-n \mathcal{P}}(x)$ denotes the element of $f^{-n \mathcal{P}}$ which contains $x$. To find an upper bound for the volume of this dynamical ball it is enough to estimate the volume of $f^{-n \mathcal{P}}(x)$ when $n$ is a hyperbolic time for $x$.

Let $P \in \mathcal{P}$ be such that $f^{-n}(P)$ has a positive Lebesgue measure subset $\tilde{P}$ of points for which $n$ is a hyperbolic time and choose $h$ such that $R_{h} \supset P$. Let $\tilde{Q} \in \mathcal{P}$ be such that $Q=\tilde{Q} \cap \tilde{P}$ has positive Lebesgue measure and choose $l$ such that $R_{l} \supset Q$.

We consider the projection of $\hat{P}=\exp _{x_{l}}^{-1}(\tilde{P})$ on $E_{x_{l}}$ parallel to $F_{x_{l}}$. Its diameter will be bounded by a constant which is a function of $f$ and $s$ only, since the number of different $R_{l}$ is finite. Projecting $\hat{Q}$ on the complementary direction $F_{x_{l}}$ parallel to $E_{x_{l}}$ we may use the backward contraction and bounded area distortion for hyperbolic times along $F$-disks to estimate the area along $F$-disks and integrate to deduce a volume estimate.

Indeed, observe that since the $E$ direction is uniformly contracted by $D f$, if we fix a point $x_{0} \in Q$, the corresponding point $x_{n}=f^{n}\left(x_{0}\right) \in P \cap f^{n}(Q)$ and a $E$-disk $\gamma$ which crosses $R_{h}$, then the connected component $\hat{\gamma}$ of $f^{-n}(\gamma) \cap R_{l}$ containing $x_{0}$ is a $E$-disk which also crosses 
$R_{l}$. Moreover distances along $\gamma$ are uniformly expanded by $f^{-1}$. Thus every point $w_{0} \in \hat{\gamma}$ is such that $w_{k}=f^{k}\left(w_{0}\right)$ and $x_{k}=f^{k}\left(x_{0}\right)$ satisfy

$$
C \frac{\delta_{1}}{4}>C s \geq \operatorname{dist}\left(w_{0}, x_{0}\right) \geq C \lambda^{-k} \operatorname{dist}\left(w_{k}, x_{k}\right),
$$

for some constant $C>0$ depending on $f$ only. Hence if we take $s$ small enough then we can ensure that $w_{k}$ is close enough to $x_{k}$ for $k=1, \ldots, n$ so that $n$ is also an hyperbolic time for all $w_{0} \in \hat{\gamma}$. Thus we can consider $F$-disks $\beta_{q}$ through the points $q$ of $Q$ parallel to $F$, which are transversal to $\hat{\gamma}$. Then the images $f^{n}\left(\beta_{q}\right)$ will be $F$-disks crossing $R_{l}$ which together cover $P \cap f^{n}(Q)$, see Figure 1 .

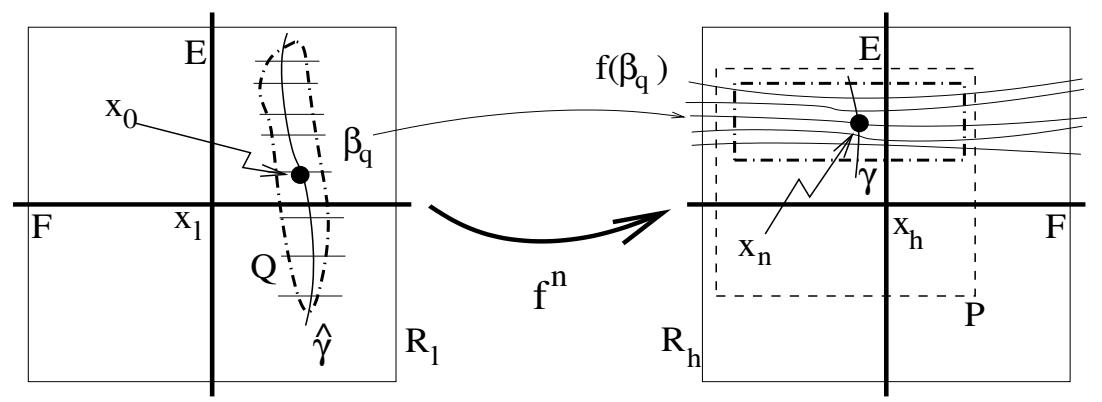

Figure 1. The diameter of the elements of $\mathcal{E}_{n}$ through the use of $E$-disks and images of $F$-disks on a hyperbolic time.

The preimages $f^{-n}\left(P \cap f^{n}(Q) \cap f^{n}\left(\beta_{q}\right)\right)$ then form a cover of $Q$ and these predisks are $F$-disks whose diameter is smaller than $e^{-c n}$.

Using Tonelli's Theorem we can write Leb $(Q)=\int_{\hat{\gamma}} m\left(Q \cap \beta_{q}\right) d q$ where $m$ denotes the $d_{F^{-}}$ dimensional Lebesgue measure induced by Leb on $F$-disks and $d q$ is Lebesgue measure along the disk $\hat{\gamma}$. By the Change of Variables Formula together with the bounded area distortion along hyperbolic times in the partially hyperbolic setting given by Proposition 3.11 we get for each $q \in \hat{\gamma}$

$$
\begin{aligned}
m\left(Q \cap \beta_{q}\right) & =\int_{\beta_{q}} \chi_{Q} d m=\int_{f^{-n}\left(f^{n}\left(\beta_{q}\right)\right)} \chi_{Q} d m \\
& =\int_{f^{n}\left(\beta_{q}\right)}\left(\chi_{Q} \circ f^{-n}\right) \cdot\left|\operatorname{det} D f^{-n}\right| f^{n}\left(\beta_{q}\right) \mid d m \\
& =\int_{f^{n}\left(\beta_{q}\right)} e^{-S_{n} J\left(f^{-n}(z)\right)} \chi_{f^{n}(Q)}(z) d m(z) \\
& \leq C_{3} \cdot e^{-S_{n} J\left(f^{-n}(q)\right)} \cdot m\left(f^{n}(Q) \cap f^{n}\left(\beta_{q}\right)\right),
\end{aligned}
$$

thus Leb $(Q) \leq \int_{\hat{\gamma}} C_{3} e^{-S_{n} J(q)} m\left(f^{n}(Q) \cap f^{n}\left(\beta_{q}\right)\right) d q$. But by (3.12) we see that every $q \in \hat{\gamma} \cap Q$ satisfies

$$
d\left(f^{k}(q), f^{k}(x)\right) \leq C \lambda^{k} \frac{\delta_{1}}{4}, \quad \text { for } \quad k=0, \ldots, n .
$$

Hence because $J$ is at least $C^{1+\alpha}$ for some $\alpha \in(0,1)$ with Hölder constant $C>0$ (in fact we can take $\alpha=1$ if $f$ is $C^{2}$ ) the usual bounded distortion argument provides a constant $C_{0}>0$ 
such that

$$
\log \frac{\left|\operatorname{det} D f^{n}\right| F_{q} \mid}{\left|\operatorname{det} D f^{n}\right| F_{x} \mid}=\sum_{j=0}^{n-1} \log \frac{\left|\operatorname{det} D f\left(f^{j}(q)\right)\right|}{\left|\operatorname{det} D f\left(f^{j}(x)\right)\right|} \leq \sum_{j=0}^{n-1} C d\left(f^{j}(q), f^{j}(x)\right)^{\alpha} \leq C_{0} .
$$

Hence $\left|S_{n} J(q)-S_{n} J(x)\right| \leq C_{0}$ and by the above integration estimates we get

$$
\operatorname{Leb}(Q) \leq \int_{\hat{\gamma}} C_{3} e^{C_{0}} e^{-S_{n} J(x)} m\left(f^{n}(Q) \cap f^{n}\left(\beta_{q}\right)\right) d q \leq \tilde{C} e^{-S_{n} J(x)},
$$

where $\tilde{C}$ is bounded by the $d_{E}$-dimensional area $A_{E}$ of $\hat{\gamma}$ (which is a function of $s<\delta_{1} / 4$ ) times a uniform bound $A_{F}$ for the $d_{F}$-dimensional area of $f^{n}\left(\beta_{q}\right)$ (which is a function of the curvature bound $C_{2}$ from Proposition 3.10 and of $\delta_{1}$, see Figure 1) multiplied by the bounded distortion constants, that is $\tilde{C} \leq C_{3} e^{C_{0}} A_{E} A_{F}$.

This shows that we have the same kind of estimate for the volume of a dynamical ball as in the local diffeomorphism case, except for a different distortion constant and the fact that the Jacobian is calculated along the $F$ direction.

3.3.3. Weak distortion estimate. If $n$ is not a hyperbolic time but $y \in B(x, n, \delta)$ satisfies $\left|S_{n} J(x)-S_{n} J(y)\right| \leq n \zeta$ for some pair $\delta, \zeta>0$, then we obtain a weak distortion property. We can now argue similarly as above, using local inverse branches in the local diffeomorphism case (with or without singularities) to obtain $\operatorname{Leb}(B(x, n, \delta)) \leq$ $C e^{n \zeta} e^{-S_{n} J(x)}$; and $\operatorname{Leb}(Q) \leq C e^{n \zeta} e^{-S_{n} J(x)}$ in the partially hyperbolic diffeomorphism case.

\section{Hyperbolic times And large Deviations}

The statements of the main theorems and corollaries are consequences of the following more abstract result.

Theorem 4.1. Let $f: M \rightarrow M$ be a local diffeomorphism outside a non-flat singular set $\mathcal{S}$ admitting $\sigma \in(0,1)$ and $b, \delta>0$ such that Lebesgue almost every point has positive density of $(\sigma, \delta, b)$-hyperbolic times. Then given $c \in \mathbb{R}$ and a continuous function $\varphi: M \rightarrow \mathbb{R}$ items (1)-(3) of Theorem A hold.

Clearly Theorem A follows from Theorem 3.2 together with Theorem 4.1. Moreover item (1) in the statement of Theorem A is just item (1) of [62, Theorem 1] so it will not be proved here.

4.1. Upper bound for local diffeomorphisms. Here we prove the upper bound in item 2 of Theorem 4.1 .

Let $\varphi: M \rightarrow \mathbb{R}$ be a fixed continuous function. Consider for $n \geq 1$ and some fixed $\varepsilon, \delta, c>0$

$$
A_{n}=A_{n}(\delta, \varepsilon)=\left\{x: \frac{1}{n} S_{n} \Delta_{\delta}(x) \leq \varepsilon\right\} \text { and } B_{n}=\left\{x: \frac{1}{n} S_{n} \varphi(x) \geq c\right\} .
$$

Since we want to bound a limit superior from above, we can assume without loss that $\operatorname{Leb}\left(A_{n} \cap\right.$ $\left.B_{n}\right)>0$ in what follows. We fix $\zeta>0$, set $\rho$ to be a positive constant function, and find $0<\delta_{0}<\delta_{1} / 4$ and a partition $\mathcal{P}$ of $M$ as in Subsection 3.1 whose diameter is smaller than $\delta_{0}$ so that ${ }^{2} y \in \mathcal{P}(x) \Longrightarrow|J(y)-J(x)|<\zeta$. Then we use Lemma 3.6 with

\footnotetext{
${ }^{2}$ Here $f$ is a local diffeomorphism $(\mathcal{S}=\emptyset)$ on a compact manifold.
} 
$m=n, E \subset U_{1} \subset A_{n} \cap B_{n}$ such that $U_{1}$ is open ${ }^{3}$ and $\operatorname{Leb}\left(\left(B_{n} \cap A_{n}\right) \backslash E\right)<\operatorname{Leb}\left(B_{n} \cap A_{n}\right) / 2 n$. Then we can find $k \geq 1$ and a family $\mathcal{U}_{n}=\mathcal{E}_{1} \cup \cdots \cup \mathcal{E}_{k}$ of hyperbolic preballs contained in $U_{1}$ satisfying

$$
\operatorname{Leb}\left(E \triangle \bigcup \mathcal{u}_{n}\right) \leq\left(1-\frac{\theta}{4}\right)^{k}<\frac{1}{2 n} \operatorname{Leb}\left(A_{n} \cap B_{n}\right) .
$$

Note that $\operatorname{Leb}\left(\left(A_{n} \cap B_{n}\right) \backslash \mathcal{U}_{n}\right) \leq \operatorname{Leb}\left(\left(A_{n} \cap B_{n}\right) \backslash E\right)+\operatorname{Leb}\left(E \backslash \mathcal{U}_{n}\right)<\frac{1}{n} \operatorname{Leb}\left(A_{n} \cap B_{n}\right)$ and so

$$
\operatorname{Leb}\left(A_{n} \cap B_{n}\right)<\frac{n}{n-1} \operatorname{Leb}\left(\mathcal{U}_{n}\right)
$$

Observe also that for any element $P \in \mathcal{E}_{i}$ there exists $x \in M$ and a hyperbolic time $h_{i}$ of $f$ for $x$ such that $P \subset B\left(x, h_{i}, \delta_{1}\right)$, by construction, where $i=1, \ldots, k_{n}$ and $n<h_{1}<\cdots<h_{k_{n}}$. Let $\mathcal{C}_{n}$ be the set of all such pairs $\left(x, h_{i}\right)$, one for each element of $\mathcal{U}_{n}$ and to simplify the notation we write $h_{n}$ for $h_{k_{n}}$.

Note that if $(x, l),\left(x^{\prime}, l^{\prime}\right) \in \mathcal{C}_{n}$ and $x^{\prime} \in \mathcal{P}^{n}(x)$, then $x^{\prime} \in B\left(x, n, 2 \delta_{0}\right)$ and $B\left(x, l, \delta_{0}\right) \cup$ $B\left(x^{\prime}, l^{\prime}, \delta_{0}\right) \subset B\left(x, n, 2 \delta_{0}\right)$. Hence we may replace every pair of elements of $\mathcal{C}_{n}$ in the same atom of $\mathcal{P}^{n}$ by one of them, obtaining a coarser cover $U_{n}$ of $E$ formed by dynamical balls centered around a reduced family $\widetilde{\mathcal{C}_{n}} \subset \mathcal{C}_{n}$ of points so that, from Subsection 3.3.3

$$
\operatorname{Leb}\left(A_{n} \cap B_{n}\right) \leq \frac{n}{n-1} \operatorname{Leb}\left(U_{n}\right) \leq \frac{C n e^{n \zeta}}{n-1} \sum\left\{e^{-S_{n} J(x)} \cdot \delta_{x}: x \in \widetilde{\mathcal{C}_{n}}\right\} .
$$

Following the arguments in the proof of $[62$, Thm.1(2)] we consider the measure

$$
\sigma_{n}=Z_{n}^{-1} \sum\left\{e^{-S_{n} J(x)} \cdot \delta_{x}: x \in \widetilde{\mathcal{C}_{n}}\right\} \quad \text { where } Z_{n}=\sum\left\{e^{-S_{n} J(x)}: x \in \widetilde{\mathcal{C}_{n}}\right\} .
$$

Note that by definition each atom of $\mathcal{P}^{n}=\bigvee_{i=0}^{n-1} f^{-i} \mathcal{P}$ contains at most one point from $\widetilde{\mathcal{C}_{n}}$. Thus using [61, Lemma 9.9] we have

$$
H_{\sigma_{n}}\left(\bigvee_{i=0}^{n-1} f^{-i} \mathcal{P}\right)-\int S_{n} J(x) d \sigma_{n}(x)=\log \sum\left\{e^{-S_{n} J(x)}: x \in \widetilde{\mathbb{C}_{n}}\right\} .
$$

Setting $\mu_{n}=n^{-1} \sum_{i=0}^{n} f_{*}^{i} \sigma_{n}$ and $\mu$ a weak* accumulation point of $\mu_{n}$, we may modify the initial partition $\mathcal{P}$ according to Proposition 3.4 and Remark 3.8 so that its diameter is smaller than $\delta_{1} / 2$ and $\mu(\partial \mathcal{P})=0$ without loss, keeping $\mathcal{C}_{n}$ unchanged. As in [61, pag. 220] from the above we can deduce that for every $q \geq 1$

$$
\begin{aligned}
\limsup _{n \rightarrow+\infty} \frac{1}{n} \log Z_{n} & \leq \frac{1}{q} \limsup _{n \rightarrow+\infty} H_{\mu_{n}}\left(\bigvee_{i=0}^{q-1} f^{-i} \mathcal{P}\right)+\limsup _{n \rightarrow+\infty} \int-J d \mu_{n} \\
& \leq h_{\mu}(f, \mathcal{P})-\int J d \mu \leq h_{\mu}(f)-\int J d \mu
\end{aligned}
$$

if $f$ is a local diffeomorphism, ensuring that $\mu$ is $f$-invariant and that $J$ is a continuous function (in this case $\mathcal{S}=\emptyset$ and $\Delta_{\delta}$ plays no role, we may take $\Delta_{\delta} \equiv 0$ and $A_{n}=M$ ).

\footnotetext{
${ }^{3}$ Since $S_{n} \varphi$ is continuous and $S_{n} \Delta_{\delta}$ is upper-semicontinuous.
} 
Observe that, since the points in $\widetilde{\mathrm{C}_{n}} \subset \mathcal{C}_{n}$ are contained in $B_{n}$ and $\mu_{n}$ is a linear convex combination of measures of the form $n^{-1} \sum_{i=0}^{n-1} \delta_{f^{i}(x)}$, we get for all $n \geq 1$

$$
\begin{aligned}
\int \varphi \mu_{n} & =\frac{1}{n} \sum_{j=0}^{n-1} \sigma_{n}\left(\varphi \circ f^{j}\right)=Z_{n}^{-1} \sum_{x \in \widetilde{\mathrm{C}_{n}}} e^{-S_{n} J(x)} \cdot \frac{1}{n} \sum_{j=0}^{n-1} \varphi\left(f^{j}(x)\right) \\
& \geq c Z_{n}^{-1} \sum\left\{e^{-S_{n} J(x)}: x \in \widetilde{\mathrm{C}_{n}}\right\}=c
\end{aligned}
$$

and hence $\int \varphi d \mu \geq c$ also because $\varphi$ is a continuous function.

Therefore we have shown that there exists $\mu \in \mathcal{M}_{f}$ such that $\int \varphi d \mu \geq c$ and

$$
\limsup _{n \rightarrow+\infty} \frac{1}{n} \log \operatorname{Leb}\left(B_{n}\right) \leq \zeta+\limsup _{n \rightarrow+\infty} \frac{1}{n} \log Z_{n} \leq \zeta+h_{\mu}(f)-\int J d \mu .
$$

Since $\zeta>0$ was arbitrary, this completes the proof of item 2 in the statement of Theorem 4.1 and Theorem A.

4.2. Upper bound for partially hyperbolic diffeomorphisms. Here we show that a bound similar to the one in item 2 of Theorem A also holds in the case of a partially hyperbolic non-uniformly expanding attracting set.

Let $f: M \rightarrow M$ be a diffeomorphism satisfying the conditions of Theorem $\mathrm{D}$, let $\varphi: M \rightarrow \mathbb{R}$ be a continuous function, fix a real number $c$ and set $J=\log |\operatorname{det} D f| F \mid$. Observe that since we have Lemma 3.12 we may argue exactly as in the previous subsection to arrive at an inequality just like (4.1).

Again as in the previous subsection we consider $\mu_{n}=\frac{1}{n} \sum_{i=0}^{n} f_{*}^{i} \sigma_{n}$ and $\mu$ a weak* accumulation point of $\mu_{n}$. We also modify the partition $\mathcal{P}$ in such a way that the boundaries of each atom have zero measure with respect to all measures $\mu$ and $\mu_{n}, n \geq 1$.

The inequality (4.1) enables us to obtain inequalities (4.3) and (4.4) exactly as before. Together with the volume estimates obtained in Subsections 3.3.2 and 3.3.3 we can then arrive also at inequality (4.2) just by using a different distortion constant and replacing the Jacobian of $f$ by the Jacobian of $f$ along the $F$ direction. Hence we obtain the upper bound given by item 2 of Theorem A also in the setting of partially hyperbolic non-uniformly expanding attracting sets. This will be very useful to deduce Theorem D in Subsection 5.1.

4.3. Upper bound with singular/critical set. To obtain an analogous result to (4.4) in the limit with a transformation $f$ with non-flat singularities, thus proving item 3 from Theorem A and Theorem 4.1, we need some extra work. We first use the slow recurrence condition as follows. Let $0 \leq \delta_{i} \leq \varepsilon_{i}$ be a sequence such that $\varepsilon_{i} \searrow 0$ satisfying (1.6) for all pairs $\left(\varepsilon_{i}, \delta_{i}\right), i \geq 2$ and also $0<|x|<\delta_{2} \Longrightarrow|x|^{-\beta} \leq-\beta \log |x|$.

Lemma 4.2. There exists $K_{0}>0$ such that for any given $k \geq 3$ and each $x \in M \backslash \mathcal{S}$ satisfying $S_{n} \Delta_{\delta_{2}}(x) \leq \varepsilon_{2}$ and $S_{n} \Delta_{\delta_{k}}(x) \leq \varepsilon_{k}$, we have $\sum_{i=0}^{n-1} d\left(x_{i}, \mathcal{S}\right)^{-\beta} \leq K_{0} n$.

Proof. We have $\sum_{i=0}^{n-1} d\left(x_{i}, \mathcal{S}\right)^{-\beta} \leq \sum_{i \in B_{1}+B_{2}+B_{3}} d\left(x_{i}, \mathcal{S}\right)^{-\beta}$ where $B_{1}=\{0 \leq i<n$ : $\left.d\left(x_{i}, \mathcal{S}\right)^{\beta}<\delta_{k}\right\}$ and $B_{2}=\left\{0 \leq i<n: \delta_{k} \leq d\left(x_{i}, \mathcal{S}\right)^{\beta}<\delta_{2}\right\}$ and also $B_{3}=\{0, \ldots, n-$ $1\} \backslash\left(B_{1}+B_{2}\right)=\left\{0 \leq i<n: d\left(x_{i}, \mathcal{S}\right)^{\beta} \geq \delta_{2}\right\}$. Then, the choice of $x \in A_{n}\left(\delta_{2}, \varepsilon_{2}\right) \cap A_{n}\left(\delta_{k}, \varepsilon_{k}\right)$ and of $\delta_{2}$, ensure that

$$
\sum_{i=0}^{n-1} \frac{1}{d\left(x_{i}, \mathcal{S}\right)^{\beta}} \leq \beta S_{n} \Delta_{\delta_{k}}(x)+\beta S_{n} \Delta_{\delta_{2}}(x)+\frac{1}{\delta_{2}^{\beta}} \# B_{3} \leq \beta n \varepsilon_{k}+\beta n \varepsilon_{2}+n / \delta_{2}^{\beta}=K_{0} n
$$


where $K_{0}=\beta \varepsilon_{2}+\beta \varepsilon_{k}+\delta_{2}^{-\beta} \leq 2 \beta \varepsilon_{2}+\delta_{2}^{-\beta}$.

Then we set $\rho(x)=\exp \Delta_{\delta}(x)$ and use the non-degeneracy condition (S3) to obtain the following.

Lemma 4.3. Given $\zeta>0$ there exists $0<\delta_{0}<\delta_{1}$ so that any partition $\mathcal{P}$ constructed as in Subsection 3.1 .1 satisfies: $y \in \mathcal{P}^{n}(x) \Longrightarrow\left|S_{n} J(y)-S_{n} J(x)\right| \leq \zeta n$ for each $n \geq 1$ and $x \in A_{n}\left(\delta_{2}, \varepsilon_{2}\right) \cap A_{n}\left(\delta_{k}, \varepsilon_{k}\right)$ for each $k \geq 1$.

Proof. The choice of $\rho$ and the construction of $\mathcal{P}$ ensures that $y \in \mathcal{P}(x) \Longrightarrow d(y, x)<$ $\rho(x) / 2<d(x, \mathcal{S}) / 2$ for all $y, x \in M$. Condition (S3) for $y \in \mathcal{P}^{n}(x)$ and $n \geq 1, x \in A_{n}\left(\delta_{2}, \varepsilon_{2}\right) \cap$ $A_{n}\left(\delta_{k}, \varepsilon_{k}\right)$ ensures

$$
\left|S_{n} J(y)-S_{n} J(x)\right| \leq \sum_{i=0}^{n-1} B \frac{d\left(y_{i}, x_{i}\right)}{d\left(x_{i}, \mathcal{S}\right)^{\beta}} \leq B \delta_{0} \sum_{i=0}^{n-1} \frac{1}{d\left(x_{i}, \mathcal{S}\right)^{\beta}} \leq B \delta_{0} K_{0} n
$$

since $y_{i}=f^{i} y, x_{i}=f^{i} x$ satisfy $y_{i} \in \mathcal{P}\left(x_{i}\right), 0 \leq i<n$ and by Lemma 4.2. To complete the proof we just have to take $0<\delta_{0}<\zeta\left(B K_{0}\right)^{-1}$.

Fixing an initial partition $\mathcal{P}$ in the conditions of Lemma 4.3, the same arguments lead us to (4.1) as in Subsection 4.1. Likewise, if $(x, l),\left(x^{\prime}, l^{\prime}\right) \in \mathcal{C}_{n}$ and $x^{\prime} \in \mathcal{P}^{n}(x)$, then $x^{\prime} \in B\left(x, n, 2 \delta_{0}\right)$ and $B\left(x, l, \delta_{0}\right) \cup B\left(x^{\prime}, l^{\prime}, \delta_{0}\right) \subset B\left(x, n, 2 \delta_{0}\right)$. Again, we replace every pair of elements of $\mathcal{C}_{n}$ in the same atom of $\mathcal{P}^{n}$ by one of them, obtaining a coarser cover $U_{n}$ of $E$ formed by dynamical balls centered around a reduced family $\widetilde{\widetilde{C}_{n}} \subset \mathcal{C}_{n}$ of points so that, from Subsection 3.3.3 we again obtain (4.2). Since the points in $\widetilde{\mathcal{C}_{n}}$ are contained in $A_{n} \cap B_{n}$, we reobtain (4.3) and (4.5), and also $\int \Delta_{\delta} d \mu_{n} \leq \varepsilon$ for every $n \geq 1$. Before we can use the same device of slightly perturbing $\mathcal{P}$ into $\mathcal{P}_{\xi, \tau}$ by Proposition 3.4, we need to show the following.

Lemma 4.4. The singular set $\mathcal{S}$ has null $\mu$-measure.

Proof. Arguing by contradiction, assume that $\mu(\mathcal{S})>0$. Then there exists $a>0$ such that $\mu(B(\mathcal{S}, \eta)) \geq a$ for all $\eta>0$. Let $\eta>0$ be chosen so that $\mu(\partial B(\mathcal{S}, \eta))=0$ and $\inf _{B(\delta, \eta)} \Delta_{\delta} \geq 4 \varepsilon / a$.

On the one hand, since $\mu$ is a weak* limit point of $\mu_{n}$, there exists $n_{0}$ such that for $n>n_{0}$ we have $\mu_{n}(B(\mathcal{S}, \eta)) \geq a / 2$. On the other hand, since $\Delta_{\delta} \geq 0$ we get by the choice of $\eta$

$$
\frac{4 \varepsilon}{a} \mu_{n}(B(\mathcal{S}, \eta)) \leq \mu_{n}\left(\Delta_{\delta} \cdot \chi_{B(\mathcal{S}, \eta)}\right) \leq \mu_{n}\left(\Delta_{\delta}\right) \leq \varepsilon
$$

where $\chi_{B(\mathcal{S}, \eta)}$ is the characteristic function of $B(\mathcal{S}, \eta)$, from which we deduce that $\mu_{n}(B(\mathcal{S}, \eta)) \leq$ $a / 4$. This contradiction shows that $\mu(\mathcal{S})=0$ and concludes the proof.

Lemma 4.5. The functions $\Delta_{\delta}, J$ and $\psi$ are $\mu$-integrable.

Proof. Let us define the sequence of functions

$$
\Delta_{\delta}^{k}=\xi_{k} \circ \Delta_{\delta} \text { where } \xi_{k}(x)=\left\{\begin{array}{ll}
k & \text { if }|x| \geq k \\
x & \text { if }|x|<k
\end{array}, k \geq 1 .\right.
$$

For $k>k_{0}$ with $k_{0}>|\log (\delta / 2)|$ and fixing $\eta>0$, since $\Delta_{\delta}^{k}$ is continuous and $\Delta_{\delta} \geq \Delta_{\delta}^{k}$ there is an integer $n_{0}$ such that for all $n>n_{0}$ we have

$$
\mu\left(\Delta_{\delta}^{k}\right) \leq \mu_{n}\left(\Delta_{\delta}^{k}\right)+\eta \leq \mu_{n}\left(\Delta_{\delta}\right)+\eta \leq \varepsilon+\eta .
$$


Since this holds for all $k \geq k_{0}$ and $\Delta_{\delta}(x) \rightarrow \infty$ when $x \rightarrow \mathcal{S}$, we have proved

$$
\int_{M \backslash \delta} \Delta_{\delta} d \mu<\infty
$$

Thus we get $\Delta_{\delta} \in L^{1}(\mu)$ since $\mu(\mathcal{S})=0$ by Lemma 4.4 .

For $J$ and $\psi$, note that by conditions (S2) and (S3) on the singular set $\mathcal{S}$ it follows that there exists a constant $\zeta>\beta$ such that on a small neighborhood $V$ of $\mathcal{S}$ we have

$$
\left|\log \left\|D f(x)^{-1}\right\|\right|+|\log | \operatorname{det} D f(x)^{-1}|| \leq \zeta|\log d(x, \mathcal{S})|
$$

and since $f$ is a local diffeomorphism on $M \backslash \mathcal{S}$, the $\mu$-integrability of $\Delta_{\delta}$ implies that of $\psi$ and $J$. This concludes the proof of the lemma.

Lemma 4.6. The measure $\mu$ is f-invariant.

Proof. Since $\mu(\mathcal{S})=0$ by Lemma 4.4, we can find a sequence $\eta_{n} \rightarrow 0$ of positive numbers such that $\mu\left(\partial B\left(\mathcal{S}, \eta_{n}\right)\right)=0$ for all $n \geq 1$ and $\mu\left(B\left(\mathcal{S}, \eta_{n}\right)\right) \rightarrow 0$ when $n \rightarrow \infty$.

Let us fix $\eta>0$ and a continuous function $h: M \rightarrow \mathbb{R}$. Take $n_{0}$ such that

$$
\mu\left(B\left(\mathcal{S}, \eta_{n}\right)\right) \cdot \sup |h|<\eta / 2
$$

for all $n>n_{0}$ and fix $n_{1}>n_{0}$ such that $\mu\left(B\left(\mathcal{S}, \eta_{n}\right)\right) / 2 \leq \mu_{n}\left(B\left(\mathcal{S}, \eta_{n}\right)\right) \leq 2 \mu\left(B\left(\mathcal{S}, \eta_{n}\right)\right)$ for all $n \geq n_{1}$. Then if $\tilde{f}$ is any continuous extension of $f \mid M \backslash B\left(\mathcal{S}, \eta_{n}\right)$ to $M$ (which always exists by Tietze Extension Theorem, see e.g. [43]) we get

$$
\int|h \circ f-h \circ \tilde{f}| d \mu_{n} \leq \sup |h| \cdot \mu_{n}\left(B\left(\mathcal{S}, \eta_{n}\right)\right)<\eta
$$

for all $n>n_{1}$. Also note that (4.7) holds with $\mu$ in the place of $\mu_{n}$. Since $h \circ \tilde{f}$ is continuous there exists $n_{2}>n_{1}$ such that $\left|\int h \circ \tilde{f} d \mu_{n}-\int h \circ \tilde{f} d \mu\right|<\eta$ for every $n>n_{2}$. Hence, for $n>n_{2}$ we obtain that $\left|\int h \circ \tilde{f} d \mu_{n}-\int h \circ \tilde{f} d \mu\right|$ is bounded from above by

$$
|\mu(h \circ f)-\mu(h \circ \tilde{f})|+\left|\mu(h \circ \tilde{f})-\mu_{n}(h \circ \tilde{f})\right|+\left|\mu_{n}(h \circ \tilde{f})-\mu_{n}(h \circ f)\right| \leq 3 \eta .
$$

Since $h$ was an arbitrary continuous function and $\eta$ was any positive number, we have shown that $f_{*} \mu_{n} \rightarrow f_{*} \mu$ in the weak* topology when $n \rightarrow \infty$. This is exactly what is needed to show that $\mu$ is $f$-invariant: $f_{*} \mu=\lim _{n} f_{*} \mu_{n}=\lim _{n}\left(\frac{1}{n} \sum_{j=0}^{n-1} f_{*}^{j} \sigma_{n}+\frac{f_{*}^{n} \sigma_{n}-\sigma_{n}}{n}\right)=\lim _{n} \mu_{n}=\mu$ in the weak* topology, concluding the proof.

Now we can use Proposition 3.4 to obtain a perturbation $\mathcal{P}_{\xi, \tau}$ of $\mathcal{P}$ ensuring the following. We consider $\tilde{J}$ a continuous extension of $J \chi_{M \backslash B(\delta, \xi)}$ to $M$ with the same range (this is Tietze's Extension Theorem) for $0<\xi<\delta$ and write

$$
\begin{aligned}
\limsup _{n \rightarrow \infty} \mu_{n}(-J) & =\limsup _{n \rightarrow \infty}\left[\mu_{n}\left((-J+\tilde{J}) \chi_{B(\delta, \xi)}\right)+\mu_{n}(-\tilde{J})\right] \\
& \leq 2 \limsup _{n \rightarrow \infty} \mu_{n}\left(\zeta \Delta_{\delta}\right)+\mu(-\tilde{J}) \leq 2 \zeta \varepsilon-\mu(\tilde{J})
\end{aligned}
$$

since $\tilde{J}$ is continuous and $|-J+\tilde{J}| \chi_{B(\mathcal{S}, \xi)} \leq 2|J| \chi_{B(\delta, \delta)} \leq 2 \zeta \Delta_{\delta}$ by (4.6). Taking $\xi \rightarrow 0$ we get $\mu(\tilde{J}) \rightarrow \mu(J)$ because $J \in L^{1}(\mu)$ and $H_{\mu}\left(\mathcal{P}_{\xi, \tau}\right)<\infty$, together with (4.3) we arrive at

$$
\limsup _{n \rightarrow+\infty} \frac{1}{n} \log Z_{n} \leq h_{\mu}\left(f, \mathcal{P}_{\xi, \tau}\right)-\int J d \mu+2 \zeta \varepsilon
$$


for some $\mu \in \mathcal{M}_{f}$ with $\mu(\varphi) \geq c$ and $\Delta_{\delta} \in L^{1}(\mu)$, which is enough to prove item (3) of Theorem 4.1 and Theorem A.

\section{Strictly Negative UPPER BOUnd}

Here we prove Theorem B and Theorem D. For a $C^{1}$ endomorphism $f$ it is known [52] that the following inequality (also known as Ruelle's inequality) holds for every $f$-invariant probability measure $\mu$

$$
h_{\mu}(f) \leq \int \Sigma^{+} d \mu
$$

where $\Sigma^{+}$denotes the sum of the positive Lyapunov exponents at $\mu$-a.e. point. In Subsection 5.3 we present a proof of this inequality in the setting of maps which are local diffeomorphisms away from a non-flat singular set $\mathcal{S}$ with zero Lebesgue measure, for invariant probability measures $\mu$ such that $\log d(x, \mathcal{S})$ is $\mu$-integrable.

We note that in [34] a similar result was proved under more general geometric assumptions but stricter analytic hypothesis, mostly due to the fact that in [34] the authors considered $M$ to be a compact metric space admitting a finite dimensional manifold $V$ as an open dense subset and $\mathcal{S}=M \backslash V$, which demands technical conditions on how the Riemannian metric on $V$ and $f$ behave (including the first and second derivatives on local charts) near $\mathcal{S}$ for the proof to work. Our conditions are similar except that we only need the transformation $f$ to be $C^{1}$ but assume that $\log d(x, \mathcal{S})$ is integrable, which is natural in our setting.

5.1. The local diffeomorphism and partially hyperbolic case. From Ruelle's Inequality (5.1) and from Subsection 3.3 it follows that we get a non-positive upper bound in item (2) of Theorem A since $\int J d \mu$ equals the sum of the Lyapunov exponents of $\mu$ [45]. Moreover let $\mu \in \mathbb{E}$ be given. Then, since we are assuming that each element in $\mathbb{E}$ is a weak expanding measure, we have

$$
\int J d \mu=h_{\mu}(f) \leq \int \Sigma^{+} d \mu \leq \int J d \mu .
$$

Hence if $\mu \in \mathcal{M}_{f}$ is not in $\mathbb{E}$ then the inequality (5.1) is strict.

To prove Theorem B we fix a continuous $\varphi: M \rightarrow \mathbb{R}$ and replace $B_{n}$ in Subsection 4.1 with

$$
B_{n}=\left\{x \in M: \inf \left\{\left|\frac{1}{n} S_{n} \varphi(x)-\eta(\varphi)\right|: \eta \in \mathbb{E}\right\}>\omega\right\}
$$

for some $\omega>0$. Then $B_{n}$ is an open subset of $M$ and we can assume without loss that $\operatorname{Leb}\left(A_{n} \cap B_{n}\right)>0$ in what follows, for otherwise the limit superior in (1.8) is smaller than any given real number and there is nothing to prove. Hence arguing as in Subsection 4.1 we obtain a measure $\nu \in \mathcal{M}_{f}$ satisfying inf $\{|\nu(\varphi)-\eta(\varphi)|: \eta \in \mathbb{E}\}>\omega$, the bound of item (3) of Theorem A and $\Delta_{\delta} \in L^{1}(\nu)$ with $\nu\left(\Delta_{\delta}\right) \leq \varepsilon$.

If $f$ is a local diffeomorphism, i.e. $\mathcal{S}=\emptyset$, then we can use the bound given by item (2) of Theorem A and it is enough to show that $h_{\nu}(f)-\nu(J)$ is strictly negative. But we cannot have $h_{\nu}(f)-\nu(J)=0$ since by construction $\nu$ is not in $\mathbb{E}$, thus $h_{\nu}(f)-\nu(J)<0$, completing the proof of Theorem B in the case of a local diffeomorphism.

For a partially hyperbolic non-uniformly expanding attracting set we obtain a negative upper bound following the same reasoning as above since we can use the same bound from item (2) of Theorem A, as shown in Subsection 4.2, and we can also apply Ruelle's Inequality. This completes the proof of Theorem D. 
5.2. The case with singular/critical set. In the case $\mathcal{S} \neq \emptyset$ we now show that the upper bound in item (3) of Theorem A must be strictly negative for some values of $\eta, \varepsilon, \delta>0$ and for some $\nu \in \mathcal{M}_{f}$. For that we argue by contradiction and take decreasing sequences $\delta_{k} \leq \varepsilon_{k}, k \geq 2$ such that $\varepsilon_{k} \searrow 0$, each pair $\left(\delta_{k}, \varepsilon_{k}\right)$ satisfies (1.6) and assume that the corresponding measures $\nu_{k}$ obtained according to the proof of Theorem A, with $B_{n}$ as in (5.2) and $A_{n}^{k}=\cap_{i=2}^{k} A_{n}\left(\delta_{i}, \varepsilon_{i}\right)=\left\{x \in M: S_{n} \Delta_{\delta_{i}} \leq n \varepsilon_{i}, i=2, \ldots, k\right\}$ in the place of $A_{n}$, for each $k \geq 2$, also satisfy

- $\nu_{k} \in \mathcal{M}_{f}, \Delta_{\delta_{i}} \in L^{1}\left(\nu_{k}\right)$ and $\nu_{k}\left(\Delta_{\delta_{i}}\right) \leq \varepsilon_{i}$ for $i=1, \ldots, k$;

- $\lim \sup _{n \rightarrow \infty} \frac{1}{n} \log \operatorname{Leb}\left(A_{n}^{k} \cap B_{n}\right) \leq h_{\nu_{k}}(f, \mathcal{P})-\int J d \nu_{k}+2 \zeta \varepsilon_{k}$;

- $h_{\nu_{k}}(f, \mathcal{P})-\int J d \nu_{k}+2 \zeta \varepsilon_{k} \geq 0$; and

- $\inf \left\{\left|\nu_{k}(\varphi)-\eta(\varphi)\right|: \eta \in \mathbb{E}\right\}>\omega$.

Above, $\mathcal{P}=\mathcal{P}_{\xi, \tau}$ is a partition obtained using Proposition 3.4 with the sequence ${ }^{4} \mu_{k}=\nu_{k}$ and $\mu$ some weak* accumulation point of the $\nu_{k}$. Thus, on the one hand, we have for any fixed $N \geq 1$

$$
h_{\nu_{k}}(f, \mathcal{P})=\inf _{j \geq 1} \frac{1}{j} H_{\nu_{k}}\left(\bigvee_{i=0}^{j-1} f^{-i \mathcal{P}}\right) \leq \frac{1}{N} H_{\nu_{k}}\left(\bigvee_{i=0}^{N-1} f^{-i} \mathcal{P}\right)
$$

and since $\mu(\partial \mathcal{P})=0$ we get

$$
\limsup _{k \rightarrow \infty} h_{\nu_{k}}(f, \mathcal{P}) \leq \frac{1}{N} H_{\mu}\left(\bigvee_{i=0}^{N-1} f^{-i} \mathcal{P}\right) .
$$

But $N \geq 1$ was arbitrarily fixed, so

$$
\limsup _{k \rightarrow \infty} h_{\nu_{k}}(f, \mathcal{P}) \leq \inf _{N \geq 1} \frac{1}{N} H_{\mu}\left(\bigvee_{i=0}^{N-1} f^{-i} \mathcal{P}\right)=h_{\mu}(f, \mathcal{P})
$$

On the other hand, choosing $J_{i}$ to be a continuous extension of $J \chi_{M \backslash B\left(\delta, \delta_{i}\right)}$ to $M$ with the same range, $i \geq 1$, we have

$$
\begin{aligned}
\limsup _{k \rightarrow \infty} \nu_{k}(-J) & =\limsup _{k \rightarrow \infty}\left[\nu_{k}\left(\left(-J+J_{i}\right) \chi_{B\left(\mathcal{S}, \delta_{i}\right)}\right)+\nu_{k}\left(-J_{i}\right)\right] \\
& \leq 2 \limsup _{k \rightarrow \infty} \nu_{k}\left(\zeta \Delta_{\delta_{i}}\right)+\mu\left(-J_{i}\right) \leq 2 \zeta \varepsilon_{i}-\mu\left(J_{i}\right)
\end{aligned}
$$

since $J_{i}$ is continuous and $\left|-J+J_{i}\right| \chi_{B\left(\mathcal{\delta}, \delta_{i}\right)} \leq 2|J| \chi_{B\left(\mathcal{S}, \delta_{i}\right)} \leq 2 \zeta \Delta_{\delta_{i}}$ by definition of $\Delta_{\delta_{i}}$ and by (4.6). Similar arguments to the ones proving Lemmas $4.4,4.5$ and 4.6 show that $J, \psi, \Delta_{\delta}$ are $\mu$-integrable and that $\mu$ is $f$-invariant. Because $i \geq 1$ can be arbitrarily chosen above and

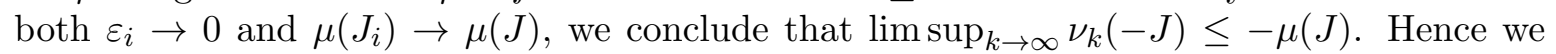
deduce

$$
0 \leq \limsup _{k \rightarrow \infty}\left(h_{\nu_{k}}(f, \mathcal{P})+\nu_{k}(-J)+2 \zeta \varepsilon_{k}\right) \leq h_{\mu}(f, \mathcal{P})-\mu(J) \leq h_{\mu}(f)-\mu(J)
$$

and also that $\inf \{|\mu(\varphi)-\eta(\varphi)|: \eta \in \mathbb{E}\} \geq \omega>0$ by construction. By Ruelle's Inequality we also get $h_{\mu}(f)-\mu(J) \leq 0$, which yields a contradiction since this means $\mu \in \mathbb{E}$. This contradiction shows that for some $k \geq 2$

$$
h_{\nu_{k}}(f, \mathcal{P})-\int J d \nu_{k}+2 \zeta \varepsilon_{k}<0
$$

\footnotetext{
${ }^{4}$ Here the independence of $K_{0}$ from $k \geq 2$ in Lemma 4.2 is crucial, allowing the size of the partition $\mathcal{P}$ not to shrink with $k$.
} 
This proves Theorem B, except for the Ruelle Inequality for maps with non-flat singularities, which is the content of the next subsection.

\subsection{Ruelle's Inequality for maps with non-flat singularities.}

Theorem 5.1. Let $f: M \backslash \mathcal{S} \rightarrow M$ be a $C^{1}$ local diffeomorphism away from a non-flat singular set $\mathcal{S}$ and $\mu$ a $f$-invariant probability measure such that $|\log d(x, \mathcal{S})|$ is $\mu$-integrable. Then

$$
h_{\mu}(f) \leq \int \Sigma^{+} d \mu
$$

where $\Sigma^{+}$denotes the sum of the positive Lyapunov exponents at a regular point, counting multiplicities.

Observe that the $\mu$-integrability of $|\log d(x, \mathcal{S})|$ implies the $\mu$-integrability of $\log ^{+}\|D f\|$, where $\log ^{+} x=\max \{0, \log x\}$, and thus the Lyapunov exponents of $f$ are well defined $\mu$ almost everywhere by Oseledets Theorem [45]. The proof we present here follows Mañé [39, Chap. IV] closely.

We start by taking the $M$ as a compact submanifold of $\mathbb{R}^{N}$ with the usual Euclidean norm and induced Riemannian structure, and considering $W_{0}$ an open normal tubular neighborhood of $M$ in $\mathbb{R}^{N}$, that is, there exists $\Phi: W_{0} \rightarrow W,(x, u) \mapsto x+u$ a $\left(C^{\infty}\right)$ diffeomorphism from a neighborhood $W_{0}$ of the zero section of the normal bundle $T M^{\perp}$ of $M$ to $W$. Let also $\pi: W \rightarrow M$ be the associated projection: $\pi(w)$ is the closest point to $w$ in $M$ for $w \in W$, so that the line through the pair of points $w, \pi(w)$ is normal to $M$ at $\pi(w)$, see e.g. [32] or [31]. Now we define for $\rho \in(0,1)$

$$
F_{0}: W_{0} \backslash\left(T_{\delta} M\right) \rightarrow W_{0}, \quad(x, u) \mapsto(f(x), \rho \cdot u)
$$

and also

$$
F: W \backslash \Phi\left(T_{\S} M\right) \rightarrow W, \quad w \mapsto\left(\Phi \circ F_{0} \circ \Phi^{-1}\right)(w) .
$$

Then clearly $F$ is a local diffeomorphism outside $\Phi\left(T_{\mathcal{S}} M\right), \overline{F(W)} \subset W$ and $M=\cap_{n \geq 0} F^{n}(W)$.

For each $n \geq 1$ consider the partition of $\mathbb{R}^{N}$ into dyadic cubes

$$
\mathcal{P}_{n}=\left\{\prod_{i=1}^{N}\left[\frac{a_{i}}{2^{n}}, \frac{a_{i}+1}{2^{n}}\right): a_{i} \in \mathbb{Z}, i=1, \ldots, N\right\} .
$$

Up to a slight translation of the partitions $\mathcal{P}_{n}$ we can assume that the probability measure $\mu$ on $M$ satisfies $\mu(M \cap \partial \mathcal{P})=0$, where $\partial \mathcal{P}=\cup_{n \geq 1} \partial \mathcal{P}_{n} \cup \mathcal{S}$. For $x \in M \backslash \partial \mathcal{P}$ we define

$$
v_{n}(x)=v_{n}^{F}(x)=\#\left\{P \in \mathcal{P}_{n}: F\left(\mathcal{P}_{n}(x)\right) \cap P \neq \emptyset\right\}
$$

and

$$
v(x)=v^{F}(x)=\limsup _{n \rightarrow \infty} v_{n}(x)
$$

where $\mathcal{P}_{n}(x)$ denotes the atom of the partition $\mathcal{P}_{n}$ containing $x$.

Lemma 5.2. Let $Q=[-1,1]^{N}$ and $x \in M \backslash \partial \mathcal{P}$. Then

$$
v(x) \leq \sup _{z \in \mathbb{R}^{n}} \#\left\{P \in \mathcal{P}_{1}:(z+D g(x) Q) \cap P \neq \emptyset\right\}
$$


Proof. For $x \in M \backslash \partial \mathcal{P}$ and $n \geq 1$ define $\varphi_{n}(y)=x+y / n$ on $\mathbb{R}^{N}$ and $W_{n}=\varphi_{n}^{-1}(W)$. Let $F_{n}: W_{n} \rightarrow F_{n}\left(W_{n}\right) \subset W_{n}$ be such that

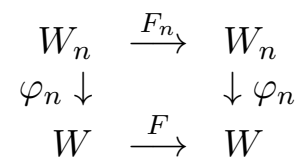

commutes. We have $F(w)=F(x)+D F(x)(w-x)+p_{x}(w)$ where $p_{x}: W \backslash \Phi\left(T_{\mathcal{S}} M\right) \rightarrow \mathbb{R}^{N}$ is $C^{1}$ and $\lim _{w \rightarrow x}\left\|p_{x}(w)\right\| /\|w-x\|=0$, where $\|\cdot\|$ is the Euclidean norm on $\mathbb{R}^{N}$. Then we write $F_{n}(y)=D F(x)(y)+q_{n}^{x}(y)+\alpha_{n}(x)$ where

$$
\alpha_{n}(x)=n \cdot F(x)-x \quad \text { and } \quad q_{n}^{x}(y)=n \cdot p_{x}(y / n+x) .
$$

Note that for $x \in M \backslash \partial \mathcal{P}$ we have $q_{n}^{x} \rightarrow 0$ uniformly on compacta. Indeed if $\|y\|<r$ for some $r>0$ there is, for each given $\delta>0$, a $n_{0} \in \mathbb{N}$ such that $\|y / n\|<\delta, \forall n \geq n_{0}$ and then, by definition of $p_{x}$, for all $\varepsilon>0$ there is $n_{1} \in \mathbb{N}$ so that $\forall n \geq n_{1},\left\|p_{x}(y / n+x)\right\|<\varepsilon\|y / n\|$ which is the same as $\left\|n \cdot p_{x}(y / n+x)\right\|<\varepsilon r$, or $\left\|q_{n}^{x}(y)\right\|<\varepsilon r$ for all sufficient large $n$.

Commutativity of the diagram implies

$$
F\left(\mathcal{P}_{n}(x)\right) \cap P \neq \emptyset \Leftrightarrow F_{n}\left(\varphi_{n}^{-1}\left(\mathcal{P}_{n}(x)\right)\right) \cap \varphi_{n}^{-1}(P) \neq \emptyset .
$$

But $\varphi_{n}^{-1}(P)$ is an element of $\mathcal{P}_{1}$ translated by some vector $y_{0} \in \mathbb{R}^{N}$. Moreover $\varphi_{n}^{-1}\left(\mathcal{P}_{n}(x)\right) \subset Q$ and so $v_{n}(x) \leq \#\left\{P \in \mathcal{P}_{1}: F_{n}(Q) \cap\left(P+y_{0}\right) \neq \emptyset\right\}$. Because $\alpha_{n}$ depends on $x$ only

$$
\begin{aligned}
v_{n}(x) & \leq \#\left\{P \in \mathcal{P}_{1}:\left(n \cdot D F(x)\left(\frac{1}{n} Q\right)+q_{n}^{x}(Q)+\alpha_{n}(x)-y_{0}\right) \cap P \neq \emptyset\right\} \\
& \leq \sup _{z \in \mathbb{R}^{N}} \#\left\{P \in \mathcal{P}_{1}:\left(D F(x) Q+q_{n}^{x}(Q)+z\right) \cap P \neq \emptyset\right\}
\end{aligned}
$$

Since $q_{n}^{x} \rightarrow 0$ on compact subsets we get

$$
\limsup _{n \rightarrow \infty} v_{n}(x) \leq \sup _{z \in \mathbb{R}^{N}} \#\left\{P \in \mathcal{P}_{1}:(D F(x) Q+z) \cap P \neq \emptyset\right\}
$$

concluding the proof of the lemma.

For the arguments which use the convergence properties of the sequence $\log v_{n}$ we need the following result.

Lemma 5.3. There exists a $\mu$-integrable function $g$ such that $0 \leq \log v_{n} \leq g$ for $\mu$-almost every point in $M$ and for all $n \geq 1$.

Proof. Fix $n \geq 1$ and consider $x \in M \backslash \partial \mathcal{P}$. On the one hand since $\mathcal{P}_{n}$ is a partition we must have $v_{n}(x) \geq 1$. On the other hand, by the bound (5.4) since the size of the edge of the cubes of $\mathcal{P}_{1}$ is $1 / 2$ in $\mathbb{R}^{N}$ we get

$$
\begin{aligned}
v_{n}(x) & \leq\left(2\left(\operatorname{diam} D F(x)(Q)+\operatorname{diam} q_{n}^{x}(Q)\right)\right)^{N} \\
\operatorname{diam} D F(x)(Q) & \leq 2 \sqrt{N} \cdot\|D F(x)\| \\
& \leq 2 \sqrt{N} \max \left\{\|D f(x)\|,\left\|D F \mid\left(T_{x} M\right)^{\perp}\right\|\right\} .
\end{aligned}
$$

Note that for $x$ far away from $\mathcal{S}$ we always get bounded expressions above since $F$ is a local diffeomorphism outside of $\Phi\left(T_{\mathcal{S}} M\right)$. To bound $\operatorname{diam} q_{n}^{x}(Q)$ we use (5.3) and consider two cases. 
First assume that $d(x, \mathcal{S}) \geq 2 / n$ and take $y \in Q$. Then for some $\theta \in[0,1]$

$$
\begin{aligned}
q_{n}^{x}(y) & =n \cdot p_{x}(y / n+x)=n \cdot(F(x+y / n)-F(x)-D F(x)(y / n)) \\
& =D F(x+\theta \cdot y / n)(y)-D F(x)(y)
\end{aligned}
$$

so we get by condition $(\mathrm{S} 1)$ on $\mathcal{S}$

$$
\begin{aligned}
\left\|q_{n}^{x}(y)\right\| & \leq \sqrt{N} \cdot(\|D F(x)\|+\|D F(x+\theta \cdot y / n)\|) \\
& \leq B \sqrt{N}\left(d(x, \mathcal{S})^{-\beta}+(d(x, \mathcal{S})-1 / n)^{-\beta}\right) \\
& \leq B \sqrt{N} \cdot d(x, \mathcal{S})^{-\beta} \cdot\left(1+2^{\beta}\right)
\end{aligned}
$$

since $1-1 /(n d(x, \mathcal{S})) \geq 1 / 2$ and $\left\|D F \mid\left(T_{x} M\right)^{\perp}\right\| \leq \rho<1 \ll d(x, \mathcal{S})^{-\beta}$ for $x$ close to $\mathcal{S}$, because $\beta>0$.

Now assume that $d(x, \mathcal{S})<2 / n$. Then we bound as follows

$$
\begin{aligned}
\left\|q_{n}^{x}(y)\right\| & \leq n \cdot\|F(x+y / n)-F(x)\|+\|D F(x)\| \cdot\|y\| \\
& \leq n \cdot \operatorname{diam} W+B \sqrt{N} \cdot d(x, \mathcal{S})^{-\beta}
\end{aligned}
$$

Hence putting (5.5), (5.6), (5.7) and (5.8) together we see that there exists a constant $\tilde{C}>0$ such that

$$
\log v_{n}(x) \leq \begin{cases}N \log \left(\tilde{C} d(x, \mathcal{S})^{-\beta}\right) & \text { if } d(x, \mathcal{S}) \geq 2 / n \\ N \log \left(\tilde{C} d(x, \mathcal{S})^{-\beta}+2 n \cdot \operatorname{diam} W\right) & \text { if } d(x, \mathcal{S})<2 / n\end{cases}
$$

But $d(x, \mathcal{S})^{-\beta}>0$ and we may assume without loss that $2 n \cdot \operatorname{diam} W \geq 2$, so

$$
\log \left(\tilde{C} d(x, \mathcal{S})^{-\beta}+2 n \cdot \operatorname{diam} W\right) \leq \log \left(\tilde{C} d(x, \mathcal{S})^{-\beta}\right)+\log (2 n \cdot \operatorname{diam} W)
$$

and if $d(x, \mathcal{S})<2 / n$ we also get

$$
\begin{aligned}
\log d(x, \mathcal{S})^{-\beta} & =-\beta \log d(x, \mathcal{S}) \geq-\beta \log (2 / n)=\beta \log (n / 2) \\
& =\beta \log (2 n \cdot \operatorname{diam} W)-\beta \log (4 \operatorname{diam} W) \quad \text { or } \\
\log (2 n \cdot \operatorname{diam} W) & \leq \log (4 \operatorname{diam} W)-\log d(x, \mathcal{S})
\end{aligned}
$$

Hence in all cases we arrive at

$$
\log v_{n}(x) \leq N \log \left(C d(x, \mathcal{S})^{-\beta}+D\right)
$$

for some positive constants $C$ and $D$. This concludes the proof.

Lemma 5.4. The following bound on the entropy holds

$$
h_{\mu}\left(f, \mathcal{P}_{n} \cap M\right)=h_{\mu}\left(F \mid M, \mathcal{P}_{n} \cap M\right) \leq \int_{M} \log v_{n}^{F} d \mu .
$$

Proof. This is [39, Lemma 12.2] without change.

Corollary 5.5. $h_{\mu}(f)=h_{\mu}(F \mid M) \leq \int_{M} \log v^{F} d \mu$.

Proof. Since $\bigvee_{n \geq 1}\left(\mathcal{P}_{n} \cap M\right)$ is the Borel $\sigma$-algebra $\mu$ mod 0 we get

$$
h_{\mu}(F \mid M)=\lim _{n \rightarrow \infty} h_{\mu}\left(F \mid M, \mathcal{P}_{n} \cap M\right) \leq \limsup _{n \rightarrow \infty} \int_{M} \log v_{n}^{F} d \mu .
$$


By Lemma 5.3 we can use the Dominated Convergence Theorem to obtain

$$
\limsup _{n \rightarrow \infty} \int_{M} \log v_{n}^{F} d \mu \leq \int_{M} \limsup \log v_{n}^{F} d \mu=\int_{M} \log v^{F} d \mu
$$

since $\log$ is monotonous increasing. This concludes the proof.

In what follows write $v^{n}(x)=v^{F^{n}}(x)$ for the analogous to $v^{F}(x)$ with $F^{n}$ in the place of $F$.

Lemma 5.6. We have

$$
h_{\mu}(f)=h_{\mu}(F \mid M) \leq \int \limsup _{n \rightarrow \infty} \frac{1}{n} \log v^{n}(x) d \mu(x) .
$$

Proof. Using [61, Thm. 4.13] and Corollary 5.5 we get for all $n \geq 1$

$$
h_{\mu}(F \mid M)=\frac{1}{n} h_{\mu}\left(F^{n} \mid M\right) \leq \int \frac{1}{n} \log v^{n}(x) d \mu(x) .
$$

Consider the sequence $g_{n}(x)=n^{-1} \log v^{n}(x)$ and observe that by Lemma 5.2 and by (5.6)

$$
\begin{aligned}
g_{n}(x) & \leq \frac{1}{n} \log \left(2 \operatorname{diam}\left(D F^{n}(x) Q\right)\right)^{N} \\
& \leq \frac{N}{n} \log (2 \sqrt{N})+\frac{N}{n} \log \left\|D F^{n}(x)\right\|=G_{n}(x) .
\end{aligned}
$$

Again by (5.6) and by definition of $F$ since $x \in M$ we get $\log \|D F(x)\| \leq \log ^{+}\|D f(x)\|$. Hence by the $f$-invariance of $\mu$ and the Sub-additive Ergodic Theorem [61, Thm. 10.1], the sequence $G_{n}(x)$ tends to a finite limit $G(x)$ for $\mu$-a.e. $x$ when $n \rightarrow \infty$.

Now by (5.10) and by Fatou's Lemma [61, Thm. 0.9]

$$
\int \liminf _{n \rightarrow \infty}\left(G_{n}-g_{n}\right) d \mu \leq \liminf _{n \rightarrow \infty} \int\left(G_{n}-g_{n}\right) d \mu .
$$

On the one hand since $\lim _{n \rightarrow \infty} G_{n}(x)$ exists $\mu$-a.e.

$$
\int \liminf _{n \rightarrow \infty}\left(G_{n}-g_{n}\right) d \mu=\int\left(G-\limsup _{n \rightarrow \infty} g_{n}\right) d \mu
$$

and, on the other hand, since $\lim _{n \rightarrow \infty} \int G_{n}(x) d \mu$ exists $\mu$-a.e. we also get

$$
\liminf _{n \rightarrow \infty} \int\left(G_{n}-g_{n}\right) d \mu=\int G d \mu-\limsup _{n \rightarrow \infty} \int g_{n} d \mu .
$$

Altogether (5.11), (5.12) and (5.13) imply

$$
\limsup _{n \rightarrow \infty} \int \frac{1}{n} \log v^{n}(x) d \mu(x) \leq \int \limsup _{n \rightarrow \infty} \frac{1}{n} \log v^{n}(x) d \mu(x)
$$

which together with (5.9) conclude the proof of the Lemma.

To finish we need to relate $\lim \sup _{n \rightarrow \infty} \frac{1}{n} \log v^{n}(x)$ with the sum of the positive Lyapunov exponents at $x$. This is done just as in [39, Chap. IV, Sec. 12] where it is proved that

$$
\limsup _{n \rightarrow \infty} \frac{1}{n} \log v^{n}(x) \leq \Sigma^{+}(x)
$$

for $\mu$-almost all $x \in M$. This together with Lemma 5.6 implies Ruelle's Inequality. The proof of Theorem 5.1 is complete. 


\section{REFERENCES}

[1] J. Alves. Statistical analysis of non-uniformly expanding dynamical systems. Publicações Matemáticas do IMPA. [IMPA Mathematical Publications]. Instituto de Matemática Pura e Aplicada (IMPA), Rio de Janeiro, 2003. XXIV Colóquio Brasileiro de Matemática. [24th Brazilian Mathematics Colloquium]. 1.1

[2] J. F. Alves. SRB measures for non-hyperbolic systems with multidimensional expansion. Ann. Sci. École Norm. Sup., 33:1-32, 2000. 1, 1, 2.4, 3

[3] J. F. Alves and V. Araujo. Random perturbations of nonuniformly expanding maps. Astérisque, 286:25-62, 2003. $1,2.4,3$

[4] J. F. Alves and V. Araújo. Hyperbolic times: frequency versus integrability. Ergodic Theory and Dynamical Systems, 24:1-18, 2004. 1

[5] J. F. Alves, V. Araújo, and B. Saussol. On the uniform hyperbolicity of nonuniformly hyperbolic systems. Proceedings of the Americam Mathematical Society, 131(4):1303-1309, 2003. 1

[6] J. F. Alves, C. Bonatti, and M. Viana. SRB measures for partially hyperbolic systems whose central direction is mostly expanding. Invent. Math., 140(2):351-398, 2000. 1, 1.1, 1.2, 2.5, 3, 3, 3.2, 3.2.4, 3.2.5

[7] J. F. Alves, S. Luzzatto, and V. Pinheiro. Markov structures and decay of correlations for non-uniformly expanding dynamical systems. Ann. Inst. H. Poincaré Anal. Non Linéaire, 22(6):817-839, 2005. 1, 2.4

[8] V. Araújo. Large deviations bound for semiflows over a non-uniformly expanding base. Bull. Braz. Math. Soc. (N.S.), 38(3):335-376, 2007. 2.2

[9] V. Araujo and M. J. Pacifico. Physical measures for infinite-modal maps. Fundamenta Mathematica, 203:211-262, 2009. 2.1

[10] V. Araújo and A. Tahzibi. Stochastic stability at the boundary of expanding maps. Nonlinearity, 18:939959, 2005. 2.3

[11] A. Arbieto and C. Matheus. Fast decay of correlations of equilibrium states of open classes of non-uniformly expanding maps and potentials. Preprint http://arxiv.org/abs/math.DS/0603629, 2006. 1, 1.4

[12] A. Arbieto, C. Matheus, S. Senti, and M. Viana. Maximal Entropy Measures for Viana Maps. Discrete and Continuous Dynamical Systems, to appear, 2007. 1.4

[13] M. Benedicks and L. Carleson. On iterations of $1-a x^{2}$ on $(-1,1)$. Annals of Math., 122:1-25, 1985. 1, 2.2

[14] M. Benedicks and L. Carleson. The dynamics of the Hénon map. Annals of Math., 133:73-169, 1991. 1

[15] M. Benedicks and L. S. Young. Absolutely continuous invariant measures and random perturbations for certain one-dimensional maps. Ergod. Th. \& Dynam. Sys., 12:13-37, 1992. 1, 1

[16] M. Benedicks and L. S. Young. SBR-measures for certain Hénon maps. Invent. Math., 112:541-576, 1993. 1

[17] M. Benedicks and L. S. Young. Markov extensions and decay of correlations for certain Hénon maps. Astérisque, 261:13-56, 2000. 1, 1

[18] C. Bonatti, L. J. Díaz, and M. Viana. Dynamics beyond uniform hyperbolicity, volume 102 of Encyclopaedia of Mathematical Sciences. Springer-Verlag, Berlin, 2005. A global geometric and probabilistic perspective, Mathematical Physics, III. 1, 1.2, 3.2.1

[19] C. Bonatti and M. Viana. SRB measures for partially hyperbolic systems whose central direction is mostly contracting. Israel J. Math., 115:157-193, 2000. 1, 2.5

[20] R. Bowen. Equilibrium states and the ergodic theory of Anosov diffeomorphisms, volume 470 of Lect. Notes in Math. Springer Verlag, 1975. 1

[21] R. Bowen and D. Ruelle. The ergodic theory of Axiom A flows. Invent. Math., 29:181-202, 1975. 1, 1

[22] L. Breiman. The individual ergodic theorem of information theory. Ann. Math. Statist., 28:809-811, 1957. 1.1

[23] M. Brin and A. Katok. Lecture Notes in Math. In Geometric dynamics (Rio de Janeiro, 1981), volume 1007, chapter On local entropy, pages 30-38. Springer, Berlin, 1983. 1.1

[24] J. Buzzi and O. Sari. Uniqueness of equilibrium measures for countable Markov shifts and multidimensional piecewise expanding maps. Ergodic Theory Dynam. Systems, 23(5):1383-1400, 2003. 1

[25] J. Buzzi, O. Sester, and M. Tsujii. Weakly expanding skew-products of quadratic maps. Ergodic Theory Dynam. Systems, 23(5):1401-1414, 2003. 2.4

[26] W. de Melo and S. van Strien. One-dimensional dynamics. Springer Verlag, 1993. 1.1, 1.1

[27] M. Denker. The central limit theorem for dynamical systems. In Dynamical Systems and Ergodic Theory, volume 23. Banach Center Publ., Warsaw, 1989. 1 
[28] M. Denker and M. Kesseböhmer. Progr. Probab. In Stochastic climate models (Chorin, 1999), volume 49, chapter Thermodynamic formalism, large deviation, and multifractals, pages 159-169. Birkhäuser, Basel, 2001. 1

[29] R. S. Ellis. Entropy, large deviations, and statistical mechanics. Reprint of the 1985 original. Classics in Mathematics. Springer-Verlag, Berlin, 2006. 1

[30] S. Gouëzel. Decay of correlations for nonuniformly expanding systems. Bull. Soc. Math. France, 134(1):131, 2006. 1, 2.4

[31] V. Guillemin and A. Pollack. Differential Topology. Prentice Hall, New Jersey, 1974. 5.3

[32] M. Hirsch. Differential Topology. Springer-Verlag, New-York, 1976. 5.3

[33] M. Jakobson. Absolutely continuous invariant measures for one-parameter families of one-dimensional maps. Comm. Math. Phys., 81:39-88, 1981. 1

[34] A. Katok and J. M. Strelcyn. Invariant manifolds, entropy and billiards. Smooth maps with singularities, volume 1222 of Lect. Notes in Math. Springer Verlag, 1986. 1.1, 5

[35] Y. Kifer. Large deviations in dynamical systems and stochastic processes. Transactions of the Americal Mathematical Society, 321(2):505-524, 1990. 1, 1.4

[36] A. Lasota and J. A. Yorke. On the existence of invariant measures for piecewise monotonic transformations. Trans. Amer. Math. Soc., 186:481-488, 1973. 2.2

[37] P. D. Liu. Pesin's Entropy Formula for Endomorphisms. Nagoya Math. J., 150:197-209, 1998. 1.3

[38] S. Luzzatto and M. Viana. Positive Lyapunov exponents for Lorenz-like families with criticalities. Astérisque, 261:201-237, 2000. Géométrie complexe et systèmes dynamiques (Orsay, 1995). 2.2

[39] R. Mañé. Ergodic theory and differentiable dynamics. Springer Verlag, New York, 1987. 2.5, 3.1.1, 3.5, 5.3, $5.3,5.3$

[40] B. McMillan. The basic theorems of information theory. Ann. Math. Statistics, 24:196-219, 1953.1 .1

[41] R. J. Metzger. Sinai-Ruelle-Bowen measures for contracting Lorenz maps and flows. Ann. Inst. H. Poincaré Anal. Non Linéaire, 17(2):247-276, 2000. 2.2

[42] L. Mora and M. Viana. Abundance of strange attractors. Acta Math., 171(1):1-71, 1993. 1

[43] J. Munkres. Topology. Prentice-Hall, New Deli, 2nd edition, 2004. 3.3.2, 4.3

[44] K. Oliveira and M. Viana. Existence and uniqueness of maximizing measures for robust classes of local diffeomorphisms. Discrete and Continuous Dynamical Systems, 15(1):225-236, 2006. 1.4, 3.1, 3.1 .2

[45] V. I. Oseledets. A multiplicative ergodic theorem: Lyapunov characteristic numbers for dynamical systems. Trans. Moscow Math. Soc., 19:197-231, 1968. 5.1, 5.3

[46] Y. Pesin and Y. Sinai. Gibbs measures for partially hyperbolic attractors. Ergod. Th. E Dynam. Sys., 2:417-438, 1982. 1, 1, 1.2

[47] V. Pinheiro. Sinai-Ruelle-Bowen measures for weakly expanding maps. Nonlinearity, 19(5):1185-1200, 2006. 1.4

[48] V. Pliss. On a conjecture due to Smale. Diff. Uravnenija, 8:262-268, 1972. 3

[49] F. Przytycki. Lyapunov characteristic exponents are nonnegative. Proceedings of the American Mathematical Society, 119(1):309-317, 1993. 2.1

[50] A. Rovella. The dynamics of perturbations of the contracting Lorenz attractor. Bull. Braz. Math. Soc., 24(2):233-259, 1993. 2.2

[51] D. Ruelle. A measure associated with Axiom A attractors. Amer. J. Math., 98:619-654, 1976. 1

[52] D. Ruelle. An inequality for the entropy of differentiable maps. Bol. Soc. Bras. Mat., 9:83-87, 1978. 1.2, 5

[53] D. Ruelle. The thermodynamical formalism for expanding maps. Comm. Math. Phys., 125:239-262, 1989. 1

[54] D. Ruelle. Thermodynamic formalism. The mathematical structures of equilibrium statistical mechanics. Cambridge Mathematical Library. Cambridge University Press, Cambridge, 2nd edition edition, 2004. 1

[55] M. Rychlik. Bounded variation and invariant measures. Studia Math., 76:69-80, 1983. 2.2

[56] C. E. Shannon. A mathematical theory of communication. The Bell System Technical Journal, 27:379-423, 1948. 1.1

[57] Y. Sinai. Gibbs measures in ergodic theory. Russian Math. Surveys, 27:21-69, 1972. 1

[58] R. Sturman and J. Stark. Semi-uniform ergodic theorems and applications to forced systems. Nonlinearity, 13:113-143, 2000. 1

[59] M. Viana. Multidimensional nonhyperbolic attractors. Inst. Hautes Études Sci. Publ. Math., 85:63-96, 1997. $1,2.4$ 
[60] M. Viana. Stochastic dynamics of deterministic systems. Publicações Matemáticas do IMPA. [IMPA Mathematical Publications]. Instituto de Matemática Pura e Aplicada (IMPA), Rio de Janeiro, 1997. XXI Coloquio Brasileiro de Matematica. [21th Brazilian Mathematics Colloquium]. 2.2

[61] P. Walters. An introduction to ergodic theory, volume 79 of Graduate Texts in Mathematics. SpringerVerlag, New York-Berlin, 1982. 4.1, 5.3, 5.3

[62] L. S. Young. Some large deviation results for dynamical systems. Trans. Amer. Math. Soc., 318(2):525-543, 1990. 1, 1.2, 4, 4.1

[63] L. S. Young. Statistical properties of dynamical systems with some hyperbolicity. Annals of Math., 147:585-650, 1998. 1, 1

\section{List OF CHANGES WITH RESPECT TO THE PUBLISHED VERSION}

Compared to Journal of Statistical Physics, Vol. 125(2):415-457, 2006 (DOI: 10.1007/s10955006-9183-y), in this version we have written in boldface the following changes: we have

(1) added the assumption that all equilibrium states $\mu \in \mathbb{E}$ are weak expanding in the statements of Theorems B and D and their Corollaries E and F.

(2) corrected comments on the examples, since some of them do not satisfy the extra assumption introduced above.

(3) added a reference to a related paper on exponentially slow approximation to the singular set of Lorenz-like maps which was published at a later date.

(4) promoted Lemma 3.4 to Proposition 3.4; strengthen its statement and provide full proof of the new statement.

(5) added Subsection 3.3.3 to state a weak bounded distortion estimate that will be used.

(6) completed the argument providing the bounds (4.1) and (4.2) in the local diffeomorphism and partial hyperbolic cases.

(7) adapted the argument in the singular/critical case in Subsection 4.3 introducing new Lemmas 4.2 and 4.3 crucially using the new Proposition 3.4 to obtain a complete proof of Theorem A.

Finally, we updated the affiliation and emails of the first author.

Vítor Araújo, Instituto de Matemática e Estatística, Universidade Federal da Bahia, Av. Ademar de Barros s/n, 40170-110 Salvador, Brazil

E-mail address: vitor.araujo.im.ufba@gmail.com and vitor.d.araujo@ufba.br

Maria José Pacifico, Instituto de Matemática, Universidade Federal do Rio de Janeiro, C.

P. 68.530, 21.945-970 Rio DE JANEIRO, BRAZIL

E-mail address: pacifico@im.ufrj.br 